Supporting Information Available

\title{
Metabolic Labeling of Mammalian Organisms with Stable Isotopes for Quantitative Proteomic Analysis
}

Christine C. Wu, Michael J. MacCoss, Kathryn E. Howell, Dwight E. Matthews, and John R. Yates, III

"To whom correspondence should be addressed. Fax: 858-784-8883. E-mail: iyates@scripps.edu 


\section{Table of Contents:}

Figure S-1: Rats appear normal and healthy during the 6 -week labeling period. Page 3

Figure S-2: Mass increase of animal is affected by access to food. Page 4 Figure S-3: Measured ${ }^{15} \mathrm{~N}$ amino acid enrichments in labeled tissue. Page 5 Figure S-4: Quantitative proteomic analysis for BiP.

Table S-1: Protein ratios for untreated-control vs. ${ }^{15} \mathrm{~N}$-enriched and cycloheximide-treated vs. ${ }^{15} \mathrm{~N}$-enriched liver samples. 


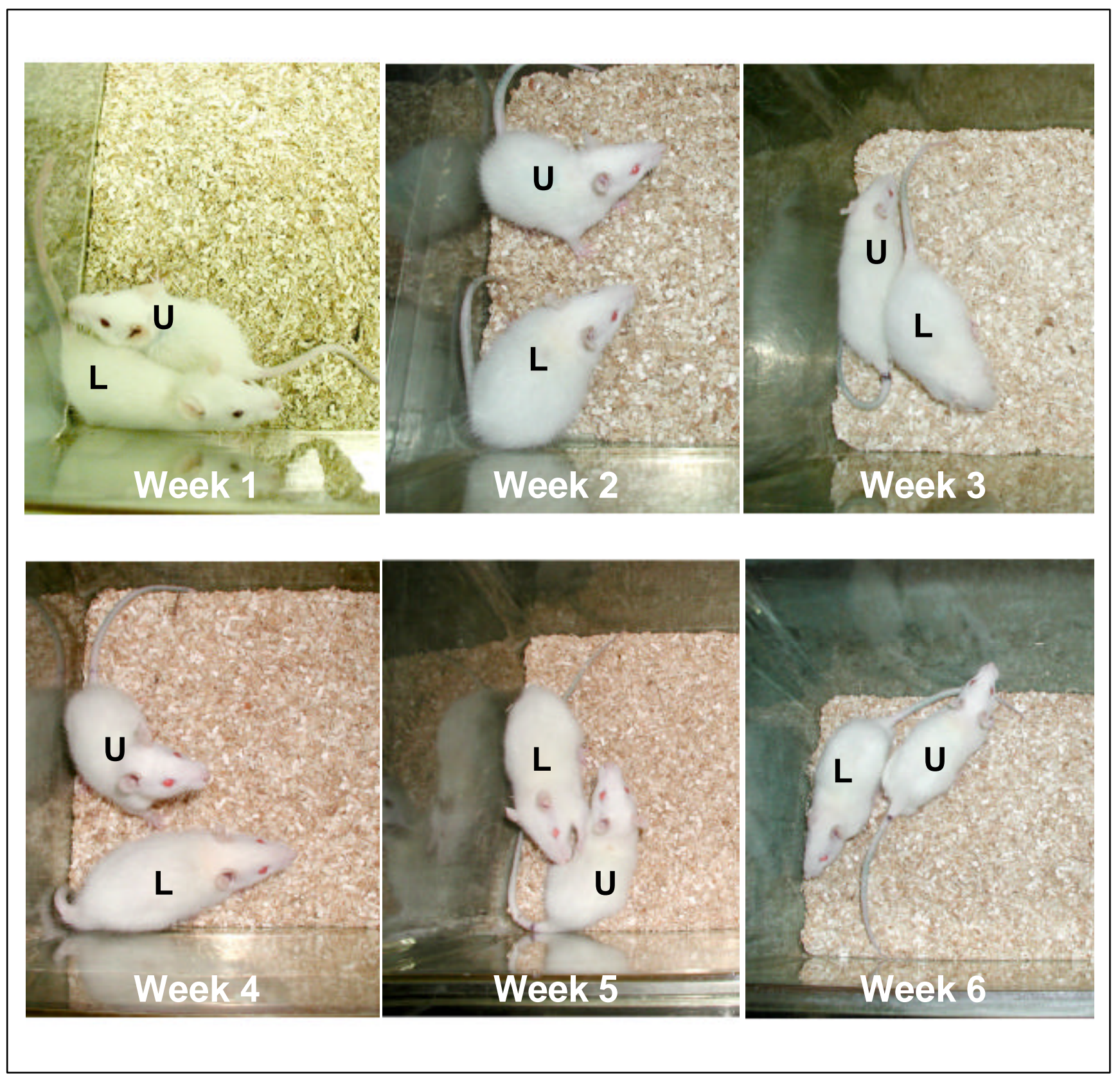

Figure S-1: Rats appear normal and healthy during the 6-week labeling period.

The labeled and unlabeled rats were photographed weekly. Both behaved similarly and underwent similar mass increases during the 6-week labeling period. 


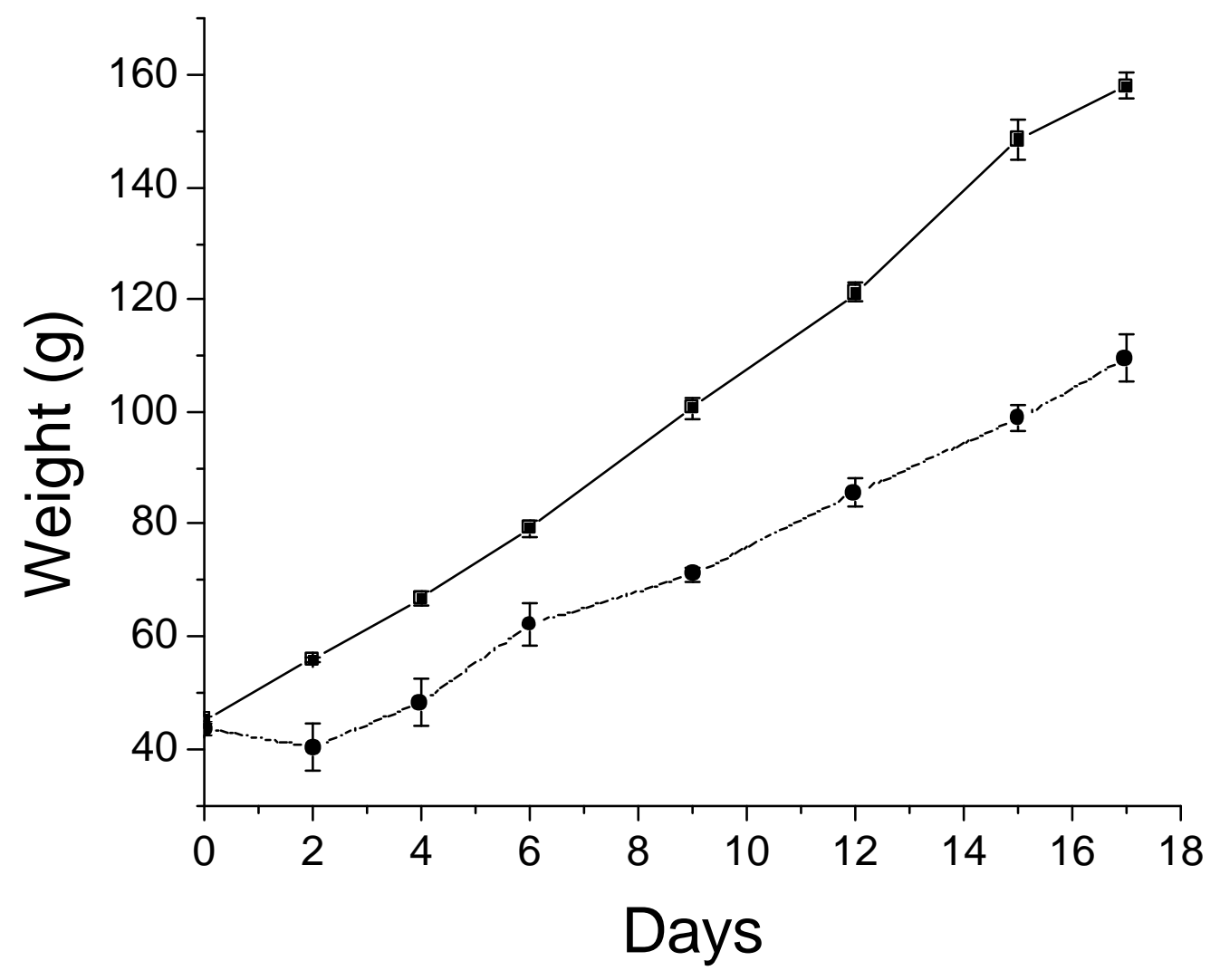

Figure S-2: Mass increase of animal is affected by access to food. Control rats were fed standard rat chow either ad libitum (line) or with scheduled feedings (4 times daily for 30-45 min) (dashed line). Limited access to food caused a 40\% decrease in mass gained during growth. 


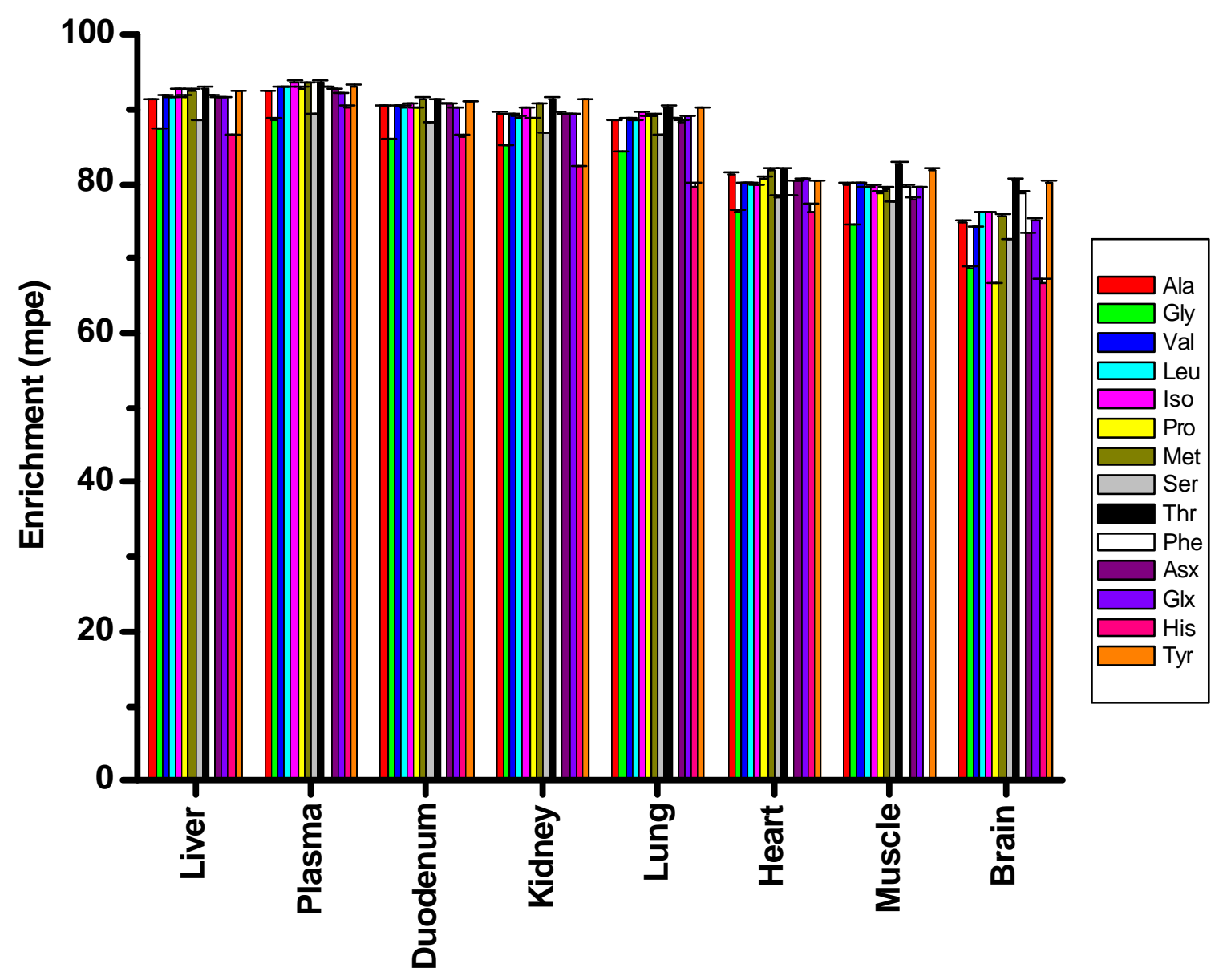

Figure S-3: Measured ${ }^{15} \mathrm{~N}$ amino acid enrichments in labeled tissue. The $M+i / M$ isotopomer ratios were measured in both unlabeled and ${ }^{15} \mathrm{~N}$-enriched tissues by gas chromatography-mass spectrometry as described in the material and methods; where $M$ is $\mathrm{m} / \mathrm{z}$ of the fragment for the natural abundance amino acid and $i$ is the number of nitrogens in the respective amino acid. Using these data, enrichments were calculated and expressed in mole percent excess (mpe). The mean \pm SD $(n=4)$ is shown for 14 amino acids in 8 different tissues. 


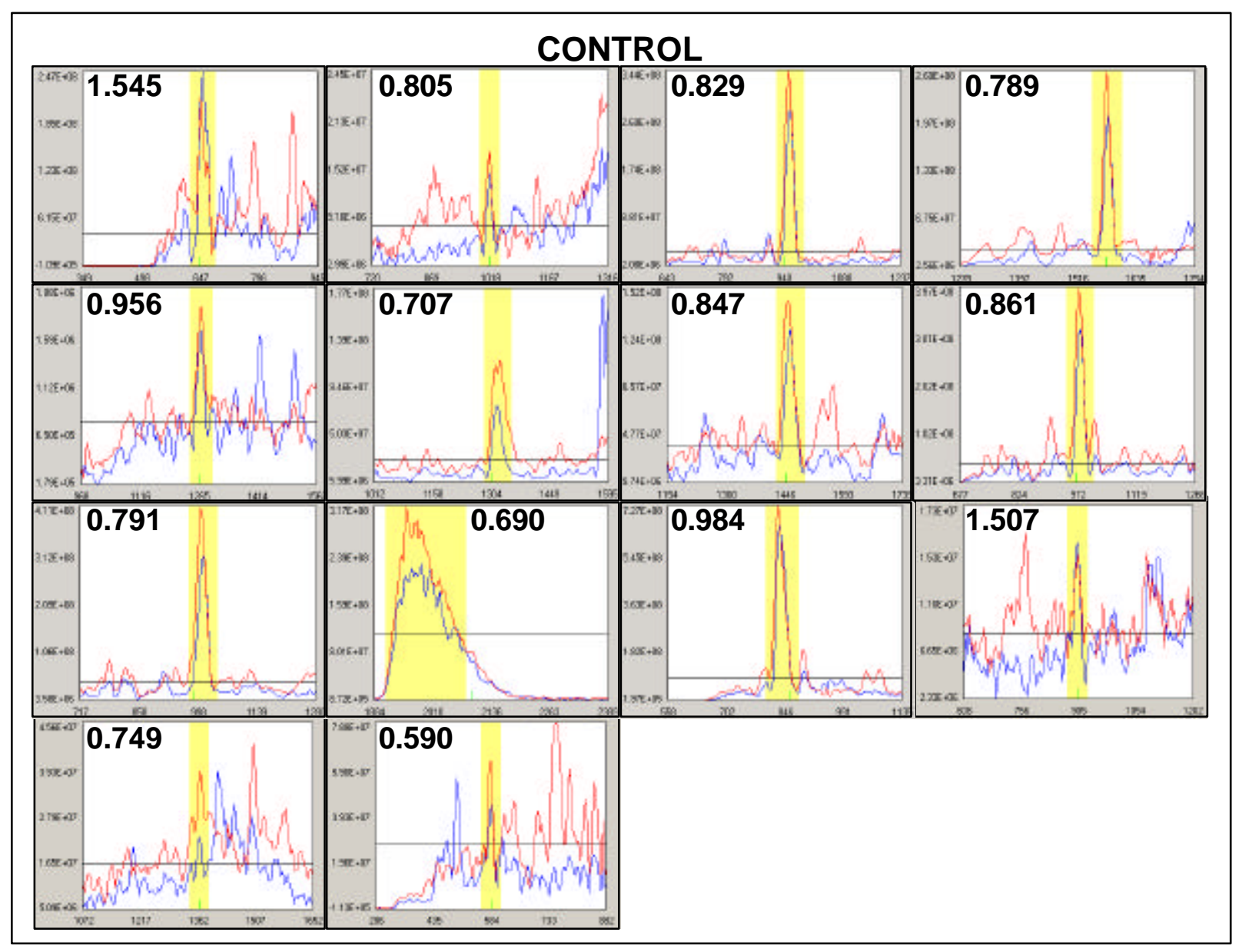

Page 6 


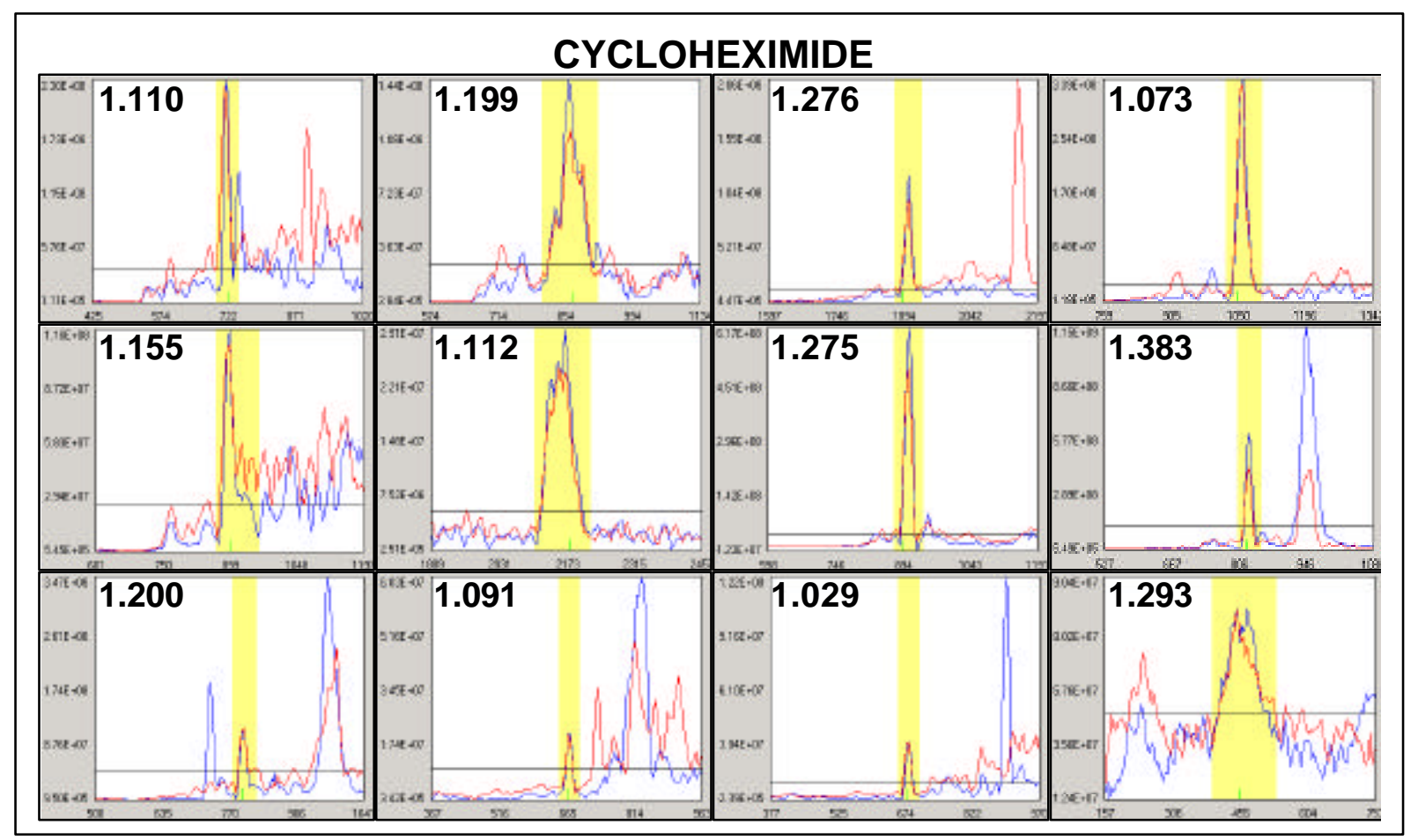

Figure S-4: Quantitative proteomic analysis for BiP. Individual extracted chromatograms for all peptides used to calculate the protein ratio for $\mathrm{BiP}(\mathrm{Hspa5}$, heat shock 70kD protein 5) (Protein \#26 in Table 1) are displayed for A) the untreated-control vs. ${ }^{15} \mathrm{~N}$-enriched and B) the cyclo heximide-treated vs. ${ }^{15} \mathrm{~N}$-enriched liver. 
2 gi|13540714|ref|NP_071796.1| plectin

12970

17.780

3 gi|13786174|ref|NP_112630.1| timeless (Drosophila) homolog

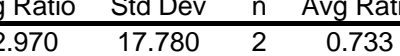

4 gi|13591977|ref|NP_112309.1| unction plakoglobin

5 gi|27720121|ref|XP 217143.1| dihydrolipoamide acetyltransferase

$\begin{array}{llll}0.641 & 0.126 & 4 & 0.049\end{array}$

$0.657 \quad 0.203$

6 gi|27690676|ref|XP 221142.1| similar to silencer-associated factor

7 gi|13928756|ref|NP_113747.1| carnitine palmitoyltransferase 1; Carnitine palmitoyltansferas alpha, liver isoform

8 gi|6981002|ref|NP_037221.1| glycogen synthase 2 (liver)

9 gi|17985951|ref|NP_444180.1| clusterin

10 gi|16758178|ref|NP 445888.1| staufen (Drosophila, RNA-binding protein)

11 gi|8392903|ref|NP_058866.1| serum amyloid P-component

12 gi|8393024|ref|NP_058690.1| complement component 3

$\begin{array}{lll}0.203 & 2 & 0.055 \\ \text { NA } & 1 & 0.056\end{array}$

$\begin{array}{llll}0.447 & \mathrm{NA} & 1 & 0.056 \\ 0.190 & 0.006 & 2 & 0.029\end{array}$

$\begin{array}{llll}0.190 & 0.006 & 2 & 0.029 \\ 0.025 & \text { NA } & 1 & 0.006\end{array}$

$\begin{array}{llll}0.392 & 0.085 & 2 & 0.111\end{array}$

Std Dev \begin{tabular}{lllll} 
NA & 1 & 0.126 & -13.082 & 0.003 \\
\hline
\end{tabular} \begin{tabular}{lllll} 
NA & 1 & 0.126 & -13.082 & 0.003 \\
NA & 1 & 0.203 & -11.945 & 0.149 \\
\hline
\end{tabular} \begin{tabular}{lllll}
0.018 & 2 & 0.018 & -7.982 & 0.021 \\
\hline
\end{tabular} \begin{tabular}{lllll} 
NA & 1 & 0.006 & -6.552 & 0.017 \\
\hline
\end{tabular} \begin{tabular}{lllll}
0.001 & 2 & 0.001 & -4.167 & 0.024 \\
\hline
\end{tabular} $\begin{array}{lllll}\text { NA } & 1 & 0.085 & -3.532 & 0.134\end{array}$

\begin{tabular}{lllllllll}
0.561 & 0.043 & 3 & 0.215 & 0.062 & 2 & 0.050 & -2.609 & 0.005 \\
1.211 & 0.001 & 2 & 0.550 & NA & 1 & 0.001 & -2.202 & 0.001 \\
\hline
\end{tabular}

\begin{tabular}{lllllllll}
1.211 & 0.001 & 2 & 0.550 & NA & 1 & 0.001 & -2.202 & 0.001 \\
\hline
\end{tabular}

\begin{tabular}{lllllllll}
1.165 & 0.498 & 4 & 0.535 & 0.223 & 3 & 0.411 & -2.178 & 0.101 \\
1.652 & 0.627 & 2 & 0.762 & 0.194 & 3 & 0.395 & -2.168 & 0.090 \\
\hline
\end{tabular} \begin{tabular}{lllllllll}
1.652 & 0.627 & 2 & 0.762 & 0.194 & 3 & 0.395 & -2.168 & 0.090 \\
\hline
\end{tabular}

\begin{tabular}{lllllllll}
0.903 & 0.230 & 7 & 0.438 & 0.277 & 4 & 0.247 & -2.062 & 0.015 \\
\hline 1.178 & 0.232 & 7 & 0.615 & 0.143 & 4 & 0.207 & -1.915 & 0.002
\end{tabular}
isoform 1; glycogen debranching enzyme; Amylo-1,6-glucosidase, 4alpha-glucanotransferase (glycogen debranching enzyme); amylo1,6-glucosidase, 4-alpha-glucanotransferase (glycogen debran...

\begin{tabular}{|c|c|c|c|c|c|c|c|c|c|c|}
\hline 14 gi|27665268|ref|XP_217054.1| & keratin 8 & 1.007 & 0.296 & 8 & 0.534 & NA & 1 & 0.296 & -1.886 & 0.003 \\
\hline 15 gi|12831225|ref|NP_075591.1| & alpha(1)-inhibitor 3, variant I & 1.305 & 0.225 & 4 & 0.700 & 0.161 & 3 & 0.202 & -1.864 & 0.011 \\
\hline 16 gi|11560087|ref|NP_071604.1| & liver glycogen phosphorylase & 0.659 & 0.133 & 8 & 0.369 & 0.200 & 14 & 0.179 & -1.786 & 0.002 \\
\hline $17 \mathrm{gi}|21955148| \mathrm{ref}|\mathrm{NP} 665725.1|$ & cytochrome P450, 3a18 & 1.176 & 0.277 & 17 & 0.661 & 0.092 & 18 & 0.204 & -1.779 & $<0.001$ \\
\hline $18 \mathrm{gi}|27676484|$ ref|XP_214876.1| & branched chain alpha-ketoacid dehydrogenase subunit E1 alpha & 0.568 & 0.038 & 2 & 0.327 & 0.083 & 2 & 0.065 & -1.737 & 0.065 \\
\hline 19 gi|19424244|ref|NP_598245.1| & brain protein 44 -like & 0.437 & 0.043 & 3 & 0.252 & 0.045 & 5 & 0.044 & -1.734 & 0.001 \\
\hline 20 gi|12025524|ref|NP_072169.1| & testis-specific histone $2 \mathrm{~b}$ & 1.289 & 0.277 & 10 & 0.745 & 0.026 & 4 & 0.240 & -1.730 & 0.002 \\
\hline 21 gi|6981260|ref|NP_037117.1| & $\begin{array}{l}\text { NADH dehydrogenase (ubiquinone) } 1 \text { alpha subcomplex } 5 \text {; NADH } \\
\text { ubiquinone oxidoreductase subunit B13 }\end{array}$ & 0.428 & NA & 1 & 0.252 & 0.049 & 4 & 0.049 & -1.698 & 0.006 \\
\hline 22 gi|16758404|ref|NP_446062.1| & peroxiredoxin 6 & 0.870 & NA & 1 & 0.546 & 0.045 & 2 & 0.045 & -1.593 & 0.062 \\
\hline 23 gi|14389299|ref|NP_112402.1| & vimentin & 0.979 & 0.020 & 2 & 0.617 & NA & 1 & 0.020 & -1.587 & 0.025 \\
\hline 24 gi|16758388|ref|NP_446054.1| & $\begin{array}{l}\text { ATP synthase, } \mathrm{H}+\text { transporting, mitochondrial F0 complex, subunit } \\
\text { F6; coupling factor } 6\end{array}$ & 0.499 & NA & 1 & 0.318 & 0.057 & 3 & 0.057 & -1.569 & 0.032 \\
\hline 25 gi|27661406|ref|XP_215139.1| & $\begin{array}{l}\text { similar to mitochondrial ribosomal protein L16; mitochondiral } \\
\text { ribosomal protein L16 }\end{array}$ & 0.445 & NA & 1 & 0.290 & 0.103 & 3 & 0.103 & -1.534 & 0.121 \\
\hline 26 gi|18777744|ref|NP_036864.2| & $\begin{array}{l}\text { lipase A, lysosomal acid; Cholesterol esterase (pancreatic), see } \\
\text { D3Wox12, D3Wox13, D3Wox26 and D3Mgh25; Cholesterol } \\
\text { esterase (pancreatic) }\end{array}$ & 1.214 & 0.066 & 2 & 0.814 & 0.357 & 2 & 0.257 & -1.491 & 0.260 \\
\hline 27 gi|13929034|ref|NP_113924.1| & $\begin{array}{l}\text { solute carrier family } 27 \text { (fatty acid transporter), member 32; solute } \\
\text { carrier family } 27 \text { (fatty acid transporter), member } 2\end{array}$ & 0.937 & 0.223 & 2 & 0.633 & 0.449 & 3 & 0.389 & -1.480 & 0.454 \\
\hline $28 \mathrm{gi}|16758014|$ ref|NP_445770.1| & hemopexin & 0.971 & 0.229 & 3 & 0.657 & NA & 1 & 0.229 & -1.478 & 0.141 \\
\hline 29 gi|6978435|ref|NP_037023.1| & $\begin{array}{l}\text { Acyl-Coa dehydrogenase, Very long chain; Very long chain Acyl- } \\
\text { Coa dehydrogenase }\end{array}$ & 0.418 & 0.054 & 3 & 0.284 & 0.090 & 5 & 0.080 & -1.472 & 0.061 \\
\hline 30 gi|6980972|ref|NP_037309.1| & $\begin{array}{l}\text { glutamate oxaloacetate transaminase 2; Glutamate oxaloacetate } \\
\text { transaminase } 2 \text {, mitochondrial (aspartate aminotransferase } 2 \text { ) }\end{array}$ & 0.468 & 0.193 & 4 & 0.320 & 0.027 & 2 & 0.168 & -1.463 & 0.366 \\
\hline $31 \mathrm{gi}|27677044|$ ref|XP_228328.1| & similar to HSP60 protein (AA 1-547) & 0.438 & 0.154 & 2 & 0.304 & NA & 1 & 0.154 & -1.441 & 0.434 \\
\hline $32 \mathrm{gi}|27662168|$ ref|XP_216944.1| & similar to cytochrome c-1 & 0.379 & 0.120 & 5 & 0.264 & 0.101 & 7 & 0.109 & -1.436 & 0.102 \\
\hline $33 \mathrm{gi}|25742657|$ ref|NP_446116.1| & sarcosine dehydrogenase & 0.606 & 0.249 & 3 & 0.423 & 0.079 & 7 & 0.142 & -1.433 & 0.099 \\
\hline 34 gi|20302075|ref|NP_620245.1| & EH-domain containing 3 & 0.950 & 0.233 & 2 & 0.667 & NA & 1 & 0.233 & -1.424 & 0.336 \\
\hline $35 \mathrm{gi}|27686093|$ ref|XP_217473.1| & similar to UDP-glucuronosyltransferase $1 \mathrm{~A} 8$ & 0.718 & 0.528 & 3 & 0.509 & 0.116 & 2 & 0.436 & -1.411 & 0.636 \\
\hline 36 gi|27675588|ref|XP_218195.1| & $\begin{array}{l}\text { similar to U2 small nuclear ribonucleoprotein auxiliary factor (65kD) } \\
\text { [Homo sapiens] }\end{array}$ & 0.781 & 0.212 & 4 & 0.555 & 0.070 & 3 & 0.170 & -1.407 & 0.142 \\
\hline 37 gi|17105350|ref|NP_476545.1| & 2,4-dienoyl CoA reductase 1 , mitochondrial & 0.273 & 0.063 & 17 & 0.195 & 0.098 & 31 & 0.087 & -1.400 & 0.005 \\
\hline
\end{tabular}




\begin{tabular}{|c|c|c|c|c|c|c|c|c|c|c|}
\hline 38 gi|27658772|ref|XP_214646.1| & $\begin{array}{l}\text { similar to vacuolar protein sorting } 35 ; \text { maternal embryonic message } \\
3 \text { [Mus musculus] }\end{array}$ & 1.216 & NA & 1 & 0.869 & 0.176 & 2 & 0.176 & -1.399 & 0.219 \\
\hline 39 gi|27711802|ref|XP_232055.1| & $\begin{array}{l}\text { similar to inner membrane protein, mitochondrial (mitofilin); motor } \\
\text { protein [Homo sapiens] }\end{array}$ & 0.290 & 0.052 & 3 & 0.213 & 0.010 & 3 & 0.037 & -1.362 & 0.065 \\
\hline 40 gi|27712654|ref|XP_232194.1| & $\begin{array}{l}\text { similar to xeroderma pigmentosum, complementation group C } \\
\text { [Homo sapiens] }\end{array}$ & 0.968 & 0.474 & 2 & 0.711 & 0.971 & 2 & 0.764 & -1.361 & 0.769 \\
\hline $\begin{array}{l}41 \text { gi|27662732|ref|XP_235480.1|, } \\
\text { gi|27690065|ref|XP_213508.1| }\end{array}$ & $\begin{array}{l}\text { similar to Probable RNA-dependent helicase p72 (DEAD-box } \\
\text { protein p72) (DEAD-box protein 17), similar to Probable RNA- } \\
\text { dependent helicase p68 (DEAD-box protein p68) (DEAD-box protein } \\
\end{array}$ & 0.616 & NA & 1 & 0.458 & 0.105 & 5 & 0.105 & -1.345 & 0.028 \\
\hline 42 gi|27661764|ref|XP_232938.1| & similar to heat shock protein 60 (liver) & 0.367 & 0.070 & 4 & 0.276 & 0.020 & 3 & 0.056 & -1.330 & 0.085 \\
\hline 43 gi|16758780|ref|NP_446356.1| & 5-oxoprolinase (ATP-hydrolysing) & 0.368 & 0.078 & 11 & 0.289 & 0.066 & 7 & 0.074 & -1.273 & 0.042 \\
\hline $44 \mathrm{gi}|13242312|$ ref|NP_077374.1| & mitochondrial aconitase (nuclear aco2 gene) & 0.205 & 0.006 & 2 & 0.161 & 0.109 & 2 & 0.077 & -1.273 & 0.626 \\
\hline $\begin{array}{l}45 \text { gi|13540640|ref|NP_110464.1|, } \\
\text { gi|18777755|ref|NP_571981.1|, } \\
\text { gi|18543335|ref|NP_570092.1|, } \\
\text { gi|13540642|ref|NP_110465.1| }\end{array}$ & $\begin{array}{l}\text { kidney specific organic anion transporter, solute carrier family } 21 \\
\text { (organic anion transporter), member } 5 \text {, kidney-specific organic } \\
\text { anion transporting polypeptide } 5 \text {; solute carrier family (organic anion } \\
\text { transporter) member } 13 \text {, organic anion transporting polypeptide } 3\end{array}$ & 0.886 & NA & 1 & 0.704 & 0.062 & 2 & 0.062 & -1.259 & 0.150 \\
\hline 46 gi|27680436|ref|XP_219392.1| & similar to hypertension-associated protein SA - rat & 0.351 & 0.046 & 6 & 0.280 & 0.031 & 6 & 0.039 & -1.254 & 0.011 \\
\hline 47 gi|18426866|ref|NP_569117.1| & $\begin{array}{l}\text { acetyl-Coenzyme A acyltransferase } 2 \text { (mitochondrial 3-oxoacyl- } \\
\text { Coenzyme A thiolase) }\end{array}$ & 0.339 & 0.065 & 5 & 0.273 & 0.078 & 8 & 0.074 & -1.242 & 0.144 \\
\hline 48 gi|16758660|ref|NP_446268.1| & calcitonin receptor & 26.830 & 20.276 & 2 & 21.982 & NA & 1 & 20.276 & -1.221 & 0.792 \\
\hline $49 \mathrm{gi}|13592145|$ ref|NP_112413.1| & malate dehydrogenase mitochondrial & 0.340 & 0.105 & 10 & 0.282 & 0.083 & 6 & 0.098 & -1.206 & 0.270 \\
\hline $50 \mathrm{gi}|27720893|$ ref $\left|X P \_217215.1\right|$ & branched chain keto acid dehydrogenase $\mathrm{E} 1$, beta polypeptide & 0.421 & NA & 1 & 0.350 & 0.048 & 3 & 0.048 & -1.203 & 0.125 \\
\hline $51 \mathrm{gi|} 27681043|\mathrm{ref}| \mathrm{XP}$ 219495.1| & similar to Tssc6 protein [Mus musculus] & 4.898 & 1.295 & 2 & 4.089 & NA & 1 & 1.295 & -1.198 & 0.539 \\
\hline $52 \mathrm{gi} 1$ 13786146|ref|NP_112615.1| & UDP-glucose dehydrogeanse & 0.375 & 0.099 & 3 & 0.314 & 0.018 & 2 & 0.081 & -1.194 & 0.472 \\
\hline 53 gi|11560024|ref|NP_071565.1| & heat shock protein 60 (liver); heat shock 60kD protein 1 & 0.345 & 0.107 & 6 & 0.289 & 0.061 & 3 & 0.096 & -1.194 & 0.437 \\
\hline $54 \mathrm{gi}|27700470|$ ref $\left|X P \_224172.1\right|$ & similar to RIKEN cDNA 5033406L14 gene [Mus musculus] & 0.274 & 0.028 & 3 & 0.231 & NA & 1 & 0.028 & -1.186 & 0.117 \\
\hline 55 gi|8393243|ref|NP_059002.1| & $\begin{array}{l}\text { dodecenoyl-Coenzyme } A \text { delta isomerase }(3,2 \text { trans-enoyl- } \\
\text { Coenyme } A \text {; dodecenoyl-Coenzyme } A \text { delta isomerase }(3,2 \text { trans- } \\
\text { enoyl-Coenyme } A \text { isomerase })\end{array}$ & 0.213 & 0.065 & 10 & 0.182 & 0.007 & 4 & 0.056 & -1.170 & 0.371 \\
\hline 56 gi|9506387|ref|NP_062236.1| & aldehyde oxidase; aldehyde oxidase (female form) & 0.440 & 0.292 & 4 & 0.376 & 0.055 & 4 & 0.210 & -1.170 & 0.682 \\
\hline 57 gi|19424338|ref|NP_598302.1| & $\begin{array}{l}\text { hydroxyacyl-Coenzyme A dehydrogenase/3-ketoacyl-Coenzyme A } \\
\text { thiolase/enoyl-Coenzyme A hydratase (trifunctional protein), beta } \\
\text { subunit }\end{array}$ & 0.294 & 0.042 & 5 & 0.252 & 0.078 & 8 & 0.067 & -1.167 & 0.296 \\
\hline $58 \mathrm{gi}|20304123|$ ref|NP_620198.1| & 3-mercaptopyruvate sulfurtransferase & 0.436 & 0.083 & 10 & 0.376 & 0.032 & 7 & 0.067 & -1.160 & 0.091 \\
\hline $59 \mathrm{gi}|27659662|$ ref $\left|X P \_214724.1\right|$ & prohibitin & 0.396 & 0.079 & 11 & 0.342 & 0.045 & 17 & 0.060 & -1.158 & 0.029 \\
\hline $60 \mathrm{gi}|16758902|$ ref|NP_446447.1| & 3-hydroxybutyrate dehydrogenase (heart, mitochondrial) & 0.488 & 0.082 & 6 & 0.425 & 0.112 & 3 & 0.092 & -1.148 & 0.363 \\
\hline 61 gi|6978441|ref|NP 037025.1| & actin, gamma 2; Actin, & 0.880 & 0.074 & 2 & 0.771 & 0.163 & 5 & 0.150 & -1.141 & 0.423 \\
\hline $62 \mathrm{gi}|21489985|$ ref|NP_659552.1| & hypothetical protein RMT-7 & 0.522 & 0.096 & 5 & 0.460 & 0.090 & 2 & 0.095 & -1.135 & 0.470 \\
\hline 63 gi|18677763|ref|NP_570839.1| & $\begin{array}{l}\text { hydroxyacyl-Coenzyme A dehydrogenase/3-ketoacyl-Coenzyme A } \\
\text { hiolase/enoyl-Coenzyme A hydratase (trifunctional protein), alpha } \\
\text { subunit }\end{array}$ & 0.281 & 0.088 & 14 & 0.249 & 0.111 & 12 & 0.099 & -1.129 & 0.420 \\
\hline 64 gi|19173766|ref|NP_596895.1| & protease, serine, 15 & 0.363 & 0.067 & 22 & 0.322 & 0.090 & 9 & 0.074 & -1.127 & 0.172 \\
\hline $\begin{array}{l}65 \text { gi|20301964|ref|NP_620189.1|, } \\
\text { gi|9507113|ref|NP_062220.1| }\end{array}$ & $\begin{array}{l}\text { Urea transporter 1-D; Urea transporter, solute carrier family } 14 \text {, } \\
\text { member } 2\end{array}$ & 0.316 & 0.047 & 2 & 0.281 & NA & 1 & 0.047 & -1.125 & 0.484 \\
\hline 66 gi|27658598|ref|XP_226269.1| & similar to ribosomal protein S18, cytosolic [validated] - rat & 0.478 & 0.138 & 7 & 0.427 & 0.006 & 2 & 0.128 & -1.119 & 0.634 \\
\hline $67 \mathrm{gi}|24025621|$ ref|NP_705886.1| & $\mathrm{Na} / \mathrm{Pi}$ cotransporter 4; sodium-phosphate cotransporter & 0.558 & 0.218 & 2 & 0.500 & NA & 1 & 0.218 & -1.116 & 0.771 \\
\hline $\begin{array}{l}68 \text { gi|27668839|ref|XP_214305.1|, } \\
\text { gi|27718097|ref|XP_235062.1|, } \\
\text { gi|27680102|ref|XP_219348.1| }\end{array}$ & $\begin{array}{l}\text { similar to genes associated with retinoid-IFN-induced mortality } 19 ; \\
\text { RIKEN cDNA } 2700054 \text { G } 14 \text { gene [Mus musculus] , similar to genes } \\
\text { associated with retinoid-IFN-induced mortality 19; RIKEN cDNA } \\
2700054 \text { G14 gene [Mus musculus] , similar to genes associated } \\
\text { with retinoid-IFN-induced mortality 19; RIKEN cDNA 2700054G14 } \\
\text { gene [Mus musculus] }\end{array}$ & 0.284 & 0.039 & 33 & 0.255 & 0.027 & 7 & 0.037 & -1.114 & 0.070 \\
\hline 69 gi|27687861|ref|XP_237585.1| & $\begin{array}{l}\text { similar to NADH-ubiquinone oxidoreductase } 24 \text { kDa subunit, } \\
\text { mitochondrial precursor }\end{array}$ & 0.320 & 0.053 & 6 & 0.288 & NA & 1 & 0.053 & -1.111 & 0.199 \\
\hline
\end{tabular}




\begin{tabular}{|c|c|c|c|c|c|c|c|c|c|c|}
\hline 70 gi|19705465|ref|NP_599192.1| & $\begin{array}{l}\text { ATP synthase, } \mathrm{H}+\text { transporting, mitochondrial F0 complex, subunit } \\
\text { b, isoform } 1\end{array}$ & 0.292 & 0.101 & 30 & 0.263 & 0.046 & 9 & 0.092 & -1.110 & 0.412 \\
\hline 71 gi|13928774|ref|NP_113758.1| & ribosomal protein S7 & 0.526 & 0.040 & 2 & 0.476 & 0.062 & 2 & 0.052 & -1.105 & 0.439 \\
\hline 72 gi|8394297|ref|NP_059003.1| & $\begin{array}{l}\text { solute carrier family } 25 \text { (mitochondrial carrier; citrate transporter) } \\
\text { precursor; citrate transport protein; mitochondrial tricarboxylate } \\
\text { carrier; solute carrier family } 25 \text { (mitochondrial carrier; citrate } \\
\text { transporter) member } 1\end{array}$ & 0.319 & 0.001 & 2 & 0.290 & 0.012 & 3 & 0.010 & -1.100 & 0.048 \\
\hline 73 gi|8392842|ref|NP_058684.1| & acid phosphatase 2; Acid phosphatase 2, lysozymal & 1.203 & 0.184 & 24 & 1.098 & 0.283 & 13 & 0.223 & -1.096 & 0.180 \\
\hline 74 gi|18959236|ref|NP_579833.1| & perosisomal 2-enoyl-CoA reductase & 0.468 & 0.019 & 3 & 0.428 & 0.005 & 2 & 0.016 & -1.093 & 0.069 \\
\hline 75 gi|27716317|ref|XP_216558.1| & $\begin{array}{l}\text { similar to succinate dehydrogenase complex, subunit B, iron sulfur } \\
\text { (Ip); iron-sulfur subunit [Homo sapiens] }\end{array}$ & 0.458 & 0.424 & 2 & 0.419 & 0.124 & 2 & 0.312 & -1.093 & 0.912 \\
\hline 76 gi|8393358|ref|NP_058701.1| & fumarate hydratase & 0.376 & 0.052 & 8 & 0.345 & 0.050 & 7 & 0.051 & -1.090 & 0.262 \\
\hline 77 gi|27684065|ref|XP_217417.1| & $\begin{array}{l}\text { similar to NADH-ubiquinone oxidoreductase } 75 \mathrm{kDa} \text { subunit, } \\
\text { mitochondrial precursor (Complex I-75Kd) (Cl-75Kd) }\end{array}$ & 0.310 & 0.043 & 4 & 0.285 & 0.021 & 2 & 0.039 & -1.088 & 0.497 \\
\hline 78 gi|8394331|ref|NP_058747.1| & $\begin{array}{l}\text { superoxide dismutase 2, mitochondrial; Superoxide dimutase 2, } \\
\text { mitochondrial }\end{array}$ & 0.415 & 0.063 & 14 & 0.382 & 0.056 & 6 & 0.061 & -1.086 & 0.283 \\
\hline 79 gi|27708576|ref|XP_216092.1| & $\begin{array}{l}\text { similar to Calcium-binding mitochondrial carrier protein Aralar2 } \\
\text { (Solute carrier family } 25, \text { member } 13 \text { ) (Citrin) }\end{array}$ & 0.501 & 0.094 & 6 & 0.466 & 0.231 & 6 & 0.176 & -1.075 & 0.738 \\
\hline $80 \mathrm{gi}|27685285| \mathrm{ref}\left|\mathrm{XP} \_220038.1\right|$ & similar to RIKEN cDNA $1810015 \mathrm{H} 18$ [Mus musculus] & 0.455 & NA & 1 & 0.425 & 0.128 & 3 & 0.128 & -1.071 & 0.724 \\
\hline $81 \mathrm{gi}|19705431|$ ref|NP_599153.1| & albumin & 0.958 & 0.149 & 16 & 0.902 & 0.073 & 4 & 0.139 & -1.062 & 0.481 \\
\hline 82 gi|18426858|ref|NP_569112.1| & succinate dehydrogenase complex, subunit $A$, flavoprotein (Fp) & 0.334 & 0.101 & 4 & 0.316 & 0.028 & 2 & 0.089 & -1.057 & 0.826 \\
\hline 83 gi|27681923|ref|XP_217339.1| & similar to heat shock protein 84 - mouse & 0.704 & 0.064 & 8 & 0.668 & 0.073 & 4 & 0.067 & -1.054 & 0.400 \\
\hline $84 \mathrm{gi}|27679580|$ ref|XP_215042.1| & $\begin{array}{l}\text { similar to ubiquinol-cytochrome-c reductase (EC 1.10.2.2) core } \\
\text { protein II precursor - rat }\end{array}$ & 0.322 & 0.008 & 2 & 0.306 & 0.006 & 2 & 0.007 & -1.052 & 0.152 \\
\hline 85 gi|11968090|ref|NP_071957.1| & short chain acyl-coenzyme A dehydrogenase & 0.328 & 0.043 & 8 & 0.315 & 0.070 & 17 & 0.063 & -1.041 & 0.635 \\
\hline 86 gi|27695144|ref|XP_214042.1| & similar to RIKEN cDNA 6030432N09 [Mus musculus] & 0.359 & 0.041 & 8 & 0.345 & 0.041 & 9 & 0.041 & -1.041 & 0.493 \\
\hline $\begin{array}{l}87 \text { gi|27706766|ref|XP_217573.1|, } \\
\text { gi|27715671|ref|XP_233365.1|, } \\
\text { gi|27708864|ref|XP_231476.1| }\end{array}$ & $\begin{array}{l}\text { ribosomal protein S4, X-linked, similar to butyrophilin-like } 2 \text { (MHC } \\
\text { class II associated) [Homo sapiens], similar to ribosomal protein } \\
\text { S4, cytosolic [validated] - rat }\end{array}$ & 0.509 & NA & 1 & 0.490 & 0.152 & 2 & 0.152 & -1.039 & 0.889 \\
\hline $88 \mathrm{gi}|27671040|$ ref|XP_224879.1| & similar to ATP synthase D chain, mitochondrial & 0.322 & 0.031 & 3 & 0.310 & 0.013 & 2 & 0.026 & -1.039 & 0.653 \\
\hline $89 \mathrm{gi}|13592103|$ ref|NP_112389.1| & sulfite oxidase & 0.342 & 0.168 & 10 & 0.332 & NA & 1 & 0.168 & -1.030 & 0.855 \\
\hline 90 gi|27706262|ref|XP_231092.1| & $\begin{array}{l}\text { similar to ASPARTATE AMINOTRANSFERASE, MITOCHONDRIAL } \\
\text { PRECURSOR (TRANSAMINASE A) (GLUTAMATE } \\
\text { OXALOACETATE TRANSAMINASE-2) }\end{array}$ & 0.433 & 0.099 & 4 & 0.421 & 0.099 & 5 & 0.099 & -1.029 & 0.862 \\
\hline $91 \mathrm{gi}|19705471|$ ref|NP_599198.1| & cold/menthol receptor 1 & 0.255 & NA & 1 & 0.248 & 0.046 & 2 & 0.046 & -1.028 & 0.865 \\
\hline 92 gi|6978705|ref|NP_037062.1| & carnitine palmitoyltransferase 2 & 0.254 & 0.002 & 2 & 0.248 & NA & 1 & 0.002 & -1.024 & 0.147 \\
\hline 93 gi|6981180|ref|NP_037330.1| & monoamine oxidase B & 0.476 & 0.074 & 34 & 0.472 & 0.091 & 32 & 0.083 & -1.008 & 0.845 \\
\hline 94 gi|16923990|ref|NP_476477.1| & Epsin 1 & 1.361 & 0.008 & 2 & 1.350 & 0.343 & 4 & 0.297 & -1.008 & 0.968 \\
\hline 95 gi|14192933|ref|NP_115792.1| & aldehyde dehydrogenase 2 ; aldehyde dehydrogenase 2 & 0.507 & 0.147 & 14 & 0.504 & 0.041 & 2 & 0.142 & -1.006 & 0.978 \\
\hline 96 gi|27720225|ref|XP_217155.1| & similar to electron transfer flavoprotein alpha chain precursor - rat & 0.369 & 0.066 & 10 & 0.367 & 0.058 & 4 & 0.064 & -1.005 & 0.959 \\
\hline $97 \mathrm{gi}|27677412|$ ref|XP_213911.1| & similar to actin gamma, cytoskeletal - rat & 0.930 & 0.162 & 15 & 0.930 & 0.245 & 5 & 0.184 & 1.000 & 1.000 \\
\hline 98 gi|11693176|ref|NP_071797.1| & acidic ribosomal protein $\mathrm{P} 0$ & 0.733 & 0.345 & 8 & 0.734 & 0.173 & 5 & 0.294 & 1.001 & 0.995 \\
\hline 99 gi|8393186|ref|NP_058768.1| & carboamyl-phosphate synthetase 1 & 0.475 & 0.107 & 59 & 0.476 & 0.083 & 42 & 0.098 & 1.002 & 0.960 \\
\hline 100 gi|13562086|ref|NP_110453.1| & glutathione peroxidase 1 & 0.945 & 0.325 & 2 & 0.950 & 0.127 & 2 & 0.247 & 1.005 & 0.986 \\
\hline 101 gi|6980956|ref|NP_036702.1| & glutamate dehydrogenase 1; Glutamate dehydrogenase & 0.319 & 0.059 & 31 & 0.323 & 0.068 & 22 & 0.063 & 1.013 & 0.820 \\
\hline 102 gi|6981312|ref|NP_037210.1| & ornithine carbamoyltransferase & 0.495 & 0.096 & 39 & 0.502 & 0.077 & 21 & 0.090 & 1.014 & 0.775 \\
\hline 103 gi|27658780|ref|XP_226345.1| & Esterase 2 & 1.328 & 0.075 & 2 & 1.355 & 0.487 & 5 & 0.437 & 1.020 & 0.944 \\
\hline 104 gi|11560046|ref|NP_071581.1| & cytochrome b5 & 1.057 & 0.240 & 20 & 1.081 & 0.357 & 5 & 0.264 & 1.023 & 0.857 \\
\hline 105 gi|27673563|ref|XP_224969.1| & $\begin{array}{l}\text { similar to solute carrier family } 25 \text { (mitochondrial carrier; ornithine } \\
\text { transporter), member } 15 \text {; solute carrier family } 25 \text { (mitochondrial } \\
\text { carrier; ornithine transporter, member 15; ornithine transporter, } \\
\text { mitochondrial } 1 \text { [Mus musculus] }\end{array}$ & 0.486 & NA & 1 & 0.498 & 0.072 & 3 & 0.072 & 1.025 & 0.800 \\
\hline 106 gi|21450773|ref|NP_114026.2| & ribosomal protein S2 & 0.660 & 0.175 & 9 & 0.684 & 0.151 & 9 & 0.163 & 1.036 & 0.759 \\
\hline
\end{tabular}


108 gi|16758036|ref|NP_445782.1| ribosomal protein L21

109 gi|6981542|ref|NP_036848.1| solute carrier 16 (monocarboxylic acid transporter), member 1 0.598

110 gi|20806145|ref|NP_620802.1| dimethylglycine dehydrogenase precursor

111 gi|6981336|ref|NP 036876.1| pyruvate carboxylase

112 gi|13540638|ref|NP_110463.1| leucyl-specific aminopeptidase PILS

$113 \mathrm{gi}|27666246| \mathrm{ref}\left|\mathrm{XP} \_234323.1\right|$ similar to ribosomal protein S7

114 gi|27721521|ref|XP_217267.1| similar to ubiquinol-cytochrome c reductase core protein I [Homo sapiens]

115 gi|12018320|ref|NP_072151.1| gamma-butyrobetaine hydroxylase

116 gi|13489075|ref|NP_071572.1| phosphodiesterase 10A; testis-specific phosphodiesterase $\quad 0.44$ PDE10A2

117 gi|8393693|ref|NP_058834.1| laminin receptor 1

$118 \mathrm{gi}|27696691| \mathrm{ref} \mid \mathrm{XP}$ 227701.1| $\quad$ similar to mannosidase, beta A, lysosomal [Mus musculus]

$\begin{array}{lll}119 \text { gil9506411|ref|NP 062256.1| } & \text { ATP synthase subunit d } & 0.267\end{array}$

\begin{tabular}{ll}
120 gi|6981010|ref|NP_037228.1| & hemoglobin, alpha 1 \\
\hline 121 gi|11120696|ref|NP_068518.1| & cysteine-sulfinate decarboxylase
\end{tabular}

122 gi|27706218|ref|XP_228468.1| similar to ACTIN, CYTOPLASMIC 1 (BETA-ACTIN)

123 gi|27690520|ref|XP_221095.1| similar to adenine nucleotide translocator

124 gi|13591997|ref|NP 112319.1| methylmalonate semialdehyde dehydrogenase gene

125 gi|27661648|ref|XP_232918.1| similar to Protein transport protein Sec24C (SEC24-related protein C)

126 gi|27712584|ref|XP_222736.1| similar to N-acetylneuraminate pyruvate lyase [Mus musculus]

127 gi|13591900|ref|NP_112265.1| 4-aminobutyrate aminotransferase

128 gi|6981360|ref|NP 036756.1 pyruvate kinase, liver and RBC

129 gi|25282449|ref|NP_742036.1| lipoprotein-binding protein \begin{tabular}{lll}
\hline 129 gi|25282449|ref|NP_742036.1| & lipoprotein-binding protein & 0.465 \\
\hline 130 gi|6981550|ref|NP_036783.1| & $\begin{array}{l}\text { solute carrier family 4, member 1, anion exchange protein 1 (kidney } \\
\text { band 3) }\end{array}$ & 1.262
\end{tabular}

131 gi|13928688|ref|NP_113697.1| glutathione S-transferase, alpha 1; Glutathione-S-transferase, alpha 0.4

\begin{tabular}{lll} 
& type (Ya) & \\
\hline 132 gi|8394283|ref|NP_058995.1| & solute carrier family 19, member 1; solute carrier family 19 & 0.515
\end{tabular} (sodium/hydrogen exchanger), member 1

133 gi|27679074|ref|XP_214532.1| mitochondrial H+-ATP synthase alpha subunit

$134 \mathrm{gi}|27663396| \mathrm{ref} \mid \mathrm{XP}$ 217005.1| similar to Protein CGI-51

\begin{tabular}{ll}
\hline 135 gil6978739|refINP 036824.1| cytochrome P450 IIA1 (hepatic steroid hydoxylas IA1) gene & 0.277
\end{tabular}

135 gi|6978739|r|

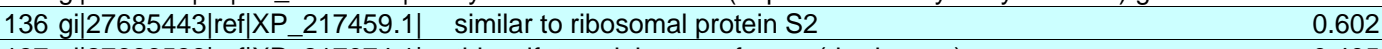

137 gi|27666538|ref|XP_217074.1| $\quad$ thiosulfate sulphurtransferase (rhodanese) 10.405

$138 \mathrm{gi} \mid$ 19424284|ref|NP_598270.1| carboxylesterase 2 (intestine, liver)

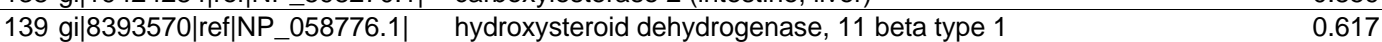

\begin{tabular}{lll}
$140 \mathrm{gi}|11560133| \mathrm{ref}\left|\mathrm{NP} \_071634.1\right|$ & alpha-tubulin & 0.632 \\
\hline $141 \mathrm{gi}|13929192| \mathrm{ref}\left|\mathrm{NP} \_114021.1\right|$ & nucleoside diphosphate kinase & 0.370
\end{tabular}

142 gi|7106240|ref|NP_037347.1| aflatoxin B1 aldehyde reductase

$143 \mathrm{gi}|11120720|$ ref|NP_068534.1| $\quad$ progesterone receptor membrane component 1; 25-Dx protein $\quad 1.058$

144 gi|13162304|ref|NP_077046.1| fatty acid amide hydrolase $\quad 0.711$

145 gi|27694044|ref|XP_227504.1| similar to 3 beta-hydroxysteroid dehydrogenase type III (3Beta-HSD 1.660 similar to 3 beta-hydroxysteroid dehydrogenase type III (3Beta-HSD
(3-beta-hydroxy-delta(5)-steroid dehydrogenase) (3-beta-hydroxy. 5-ene steroid dehydrogenase) (Progesterone reductase)

146 gi|16758586|ref|NP_446204.1| succinate-CoA ligase, GDP-forming, alpha subunit

\begin{tabular}{lcccccccc}
0.515 & NA & 1 & 0.603 & 0.089 & 2 & 0.089 & 1.171 & 0.395 \\
& & & & & & & & \\
0.254 & 0.101 & 13 & 0.298 & 0.083 & 6 & 0.096 & 1.173 & 0.366 \\
0.277 & NA & 1 & 0.326 & 0.040 & 5 & 0.040 & 1.177 & 0.052 \\
\hline 0.328 & 0.047 & 2 & 0.390 & NA & 1 & 0.047 & 1.189 & 0.313 \\
0.602 & 0.080 & 7 & 0.720 & 0.050 & 3 & 0.074 & 1.196 & 0.049 \\
0.405 & 0.062 & 15 & 0.492 & 0.083 & 5 & 0.067 & 1.215 & 0.022 \\
\hline 0.859 & 0.459 & 2 & 1.047 & NA & 1 & 0.459 & 1.219 & 0.666 \\
0.617 & 0.196 & 9 & 0.756 & 0.413 & 3 & 0.255 & 1.225 & 0.432 \\
0.632 & NA & 1 & 0.776 & 0.095 & 3 & 0.095 & 1.228 & 0.120 \\
0.370 & 0.058 & 10 & 0.455 & 0.040 & 3 & 0.055 & 1.230 & 0.039 \\
0.110 & 0.035 & 7 & 0.136 & 0.018 & 2 & 0.033 & 1.236 & 0.360 \\
1.058 & 0.100 & 2 & 1.309 & 0.233 & 3 & 0.199 & 1.237 & 0.261 \\
0.711 & 0.107 & 11 & 0.881 & 0.193 & 20 & 0.168 & 1.239 & 0.012 \\
\hline 1.660 & 0.138 & 4 & 2.070 & 0.254 & 4 & 0.204 & 1.247 & 0.030 \\
& & & & & & & & \\
& & & & & & & & \\
0.271 & 0.162 & 5 & 0.338 & 0.107 & 3 & 0.146 & 1.247 & 0.553 \\
0.638 & 0.243 & 4 & 0.801 & 0.155 & 4 & 0.204 & 1.255 & 0.301 \\
& & & & & & & &
\end{tabular}

147 gi|27674591|ref|XP_214147.1| similar to 26S protease regulatory subunit S10B (Proteasome

$\begin{array}{cccccccc}0.255 & 2 & 0.624 & 0.104 & 2 & 0.195 & 1.043 & 0.906 \\ 0.081 & 4 & 0.585 & 0.100 & 9 & 0.095 & 1.045 & 0.671 \\ 0.148 & 8 & 0.502 & 0.168 & 6 & 0.157 & 1.048 & 0.790 \\ 0.062 & 43 & 0.321 & 0.072 & 54 & 0.068 & 1.052 & 0.251 \\ \text { NA } & 1 & 0.651 & 0.755 & 2 & 0.755 & 1.060 & 0.956 \\ 0.108 & 4 & 0.611 & 0.053 & 3 & 0.090 & 1.063 & 0.623 \\ 0.043 & 5 & 0.363 & \text { NA } & 1 & 0.043 & 1.071 & 0.280\end{array}$

\begin{tabular}{llllllll} 
NA & 1 & 0.476 & 0.038 & 2 & 0.038 & 1.072 & 0.445 \\
\hline
\end{tabular}

\begin{tabular}{llllllll}
0.070 & 3 & 0.616 & 0.037 & 4 & 0.053 & 1.075 & 0.335 \\
\hline 0.355 & 7 & 1.399 & 0.650 & 4 & 0.474 & 1.077 & 0.744
\end{tabular}

\begin{tabular}{llllllll}
0.070 & 3 & 0.616 & 0.037 & 4 & 0.053 & 1.075 & 0.335 \\
0.355 & 7 & 1.399 & 0.650 & 4 & 0.474 & 1.077 & 0.744 \\
\hline
\end{tabular}

\begin{tabular}{llllllll}
0.037 & 13 & 0.290 & 0.028 & 2 & 0.036 & 1.086 & 0.420 \\
\hline
\end{tabular}

\begin{tabular}{llllllll}
0.099 & 4 & 0.474 & 0.011 & 2 & 0.086 & 1.107 & 0.570 \\
\hline
\end{tabular}

\begin{tabular}{llllllll}
0.384 & 3 & 0.731 & 0.137 & 3 & 0.288 & 1.108 & 0.778 \\
\hline NA & 1 & 1.040 & 0.057 & 2 & 0.057 & 1.112 & 0.233
\end{tabular}

\begin{tabular}{llllllll} 
NA & 1 & 1.040 & 0.057 & 2 & 0.057 & 1.112 & 0.233 \\
\hline
\end{tabular}

\begin{tabular}{lllllllll}
0.335 & 0.032 & 3 & 0.373 & 0.033 & 2 & 0.032 & 1.113 & 0.288 \\
\hline
\end{tabular}

\begin{tabular}{llllllll}
0.082 & 16 & 0.408 & 0.090 & 10 & 0.085 & 1.118 & 0.222 \\
\hline
\end{tabular}

\begin{tabular}{lllllllll}
0.675 & 0.013 & 2 & 0.761 & NA & 1 & 0.013 & 1.127 & 0.068 \\
\hline
\end{tabular}

\begin{tabular}{ccccccccc}
388 & 0.039 & 8 & 0.741 & $N A$ & 1 & 0.013 & 1.127 & 0.068 \\
\hline
\end{tabular}

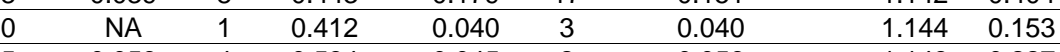

$\begin{array}{llllllll}0.059 & 4 & 0.534 & 0.045 & 2 & 0.056 & 1.148 & 0.227 \\ 0.182 & 3 & 1.460 & 0.920 & 2 & 0.552 & 1.157 & 0.720\end{array}$

$\begin{array}{llllllll}0.024 & 5 & 0.474 & 0.076 & 9 & 0.064 & 1.170 & 0.076\end{array}$

subunit $\mathrm{p} 42$ ) (p44) (Conserved ATPase domain protein 44) (CADp44) 


\begin{tabular}{|c|c|c|c|c|c|c|c|c|c|c|}
\hline 148 gi|27710460|ref|XP_213817.1| & $\begin{array}{l}\text { ATP synthase, } \mathrm{H}+\text { transporting, mitochondrial F1 complex, beta } \\
\text { polypeptide }\end{array}$ & 0.313 & 0.104 & 17 & 0.393 & 0.376 & 18 & 0.279 & 1.256 & 0.403 \\
\hline 149 gi|27694935|ref|XP_227580.1| & similar to RIKEN cDNA 1110004G14 [Mus musculus] & 0.778 & 0.147 & 5 & 0.977 & 0.404 & 4 & 0.287 & 1.256 & 0.335 \\
\hline 150 gi|6981142|ref|NP $037106.1 \mid$ & laminin chain beta 2 & 0.471 & NA & 1 & 0.592 & 0.023 & 2 & 0.023 & 1.257 & 0.085 \\
\hline $151 \mathrm{gi}|11024680|$ ref|NP_067608.1| & GERp95 & 0.688 & 0.469 & 2 & 0.867 & 0.345 & 3 & 0.391 & 1.260 & 0.650 \\
\hline 152 gi|6981330|ref|NP $036751.1 \mid$ & phenylalanine hydroxylase & 0.455 & 0.110 & 9 & 0.574 & 0.069 & 5 & 0.098 & 1.262 & 0.051 \\
\hline 153 gi|18373323|ref|NP $476446.1 \mid$ & UDP glycosyltransferase 1 family, polypeptide A6 & 0.315 & 0.043 & 3 & 0.398 & 0.170 & 7 & 0.149 & 1.263 & 0.442 \\
\hline $154 \mathrm{gi|} 27666250 \mid$ ref|XP_234325.1| & similar to putative alpha-6-fucosyltransferase [Xenopus laevis] & 0.872 & 0.055 & 3 & 1.102 & NA & 1 & 0.055 & 1.264 & 0.019 \\
\hline 155 gi|11560095|ref|NP_071609.1| & $\begin{array}{l}\text { aldehyde dehydrogenase family } 9 \text {, subfamily } \mathrm{A} 1 ; 4- \\
\text { trimethylaminobutyraldehyde dehydrogenase }\end{array}$ & 0.619 & 0.127 & 10 & 0.788 & 0.114 & 4 & 0.124 & 1.273 & 0.040 \\
\hline 156 gi|8394328|ref|NP_058746.1| & superoxide dismutase 1 , soluble; Superoxide dimutase 1 , soluble & 0.540 & 0.152 & 6 & 0.695 & NA & 1 & 0.152 & 1.287 & 0.055 \\
\hline 157 gi|11968092|ref|NP_071958.1| & dopa/tyrosine sulfotransferase & 0.539 & 0.012 & 3 & 0.696 & 0.067 & 4 & 0.052 & 1.291 & 0.011 \\
\hline 158 gi|9506497|ref|NP_062172.1| & clathrin, heavy polypeptide $(\mathrm{Hc})$ & 1.083 & 0.405 & 9 & 1.404 & 0.002 & 2 & 0.382 & 1.296 & 0.310 \\
\hline 159 gi|14010881/ref|NP_114186.1| & UDP-glucuronosyltransferase & 1.124 & 0.300 & 18 & 1.464 & 0.293 & 13 & 0.297 & 1.302 & 0.004 \\
\hline 160 gi|6978847|ref|NP $0 \overline{036924.1 \mid}$ & flavin-containing monooxygenase 1 & 1.108 & 0.345 & 17 & 1.445 & 0.431 & 20 & 0.394 & 1.304 & 0.014 \\
\hline $161 \mathrm{gi|} 21955142 \mid$ ref|NP $665722.1 \mid$ & pregnancy-zone protein & 1.000 & 0.256 & 6 & 1.305 & 0.340 & 17 & 0.322 & 1.305 & 0.059 \\
\hline 162 gi|6978871|ref|NP_036695.1| & glutamate decarboxylase 2 (islet) & 0.477 & NA & 1 & 0.624 & 0.021 & 5 & 0.021 & 1.308 & $<0.001$ \\
\hline 163 gi|25742763|ref|NP_037215.1| & heat shock $70 \mathrm{kD}$ protein 5 & 0.904 & 0.282 & 14 & 1.184 & 0.107 & 12 & 0.220 & 1.310 & 0.004 \\
\hline 164 gi|27678778|ref|XP 215029.1| & similar to epsilon 1 globin & 0.378 & 0.100 & 3 & 0.499 & 0.201 & 3 & 0.159 & 1.320 & 0.403 \\
\hline $165 \mathrm{gi}|12083649|$ ref|NP_073185.1| & p105 coactivator & 0.565 & 0.078 & 5 & 0.746 & 0.160 & 2 & 0.100 & 1.320 & 0.083 \\
\hline 166 gi|9665225|ref|NP_062569.1| & cytochrome P450 4F1 & 1.117 & 0.308 & 10 & 1.476 & 0.562 & 6 & 0.417 & 1.321 & 0.118 \\
\hline 167 gi|8393755|ref|NP_058945.1| & membrane bound $\mathrm{C} 2$ domain containing protein & 0.789 & 0.186 & 8 & 1.045 & 0.210 & 11 & 0.200 & 1.324 & 0.014 \\
\hline 168 gi|27675394|ref|XP_214050.1| & $\begin{array}{l}\text { similar to Cytosol aminopeptidase (Leucine aminopeptidase) (LAP) } \\
\text { (Leucyl aminopeptidase) (Proline aminopeptidase) (Prolyl } \\
\text { aminopeptidase) }\end{array}$ & 0.335 & 0.097 & 5 & 0.444 & 0.057 & 6 & 0.077 & 1.325 & 0.045 \\
\hline 169 gi|13591981|ref|NP_112311.1| & 2,3-oxidosqualene: lanosterol cyclase & 0.422 & 0.054 & 6 & 0.564 & 0.030 & 4 & 0.046 & 1.336 & 0.001 \\
\hline 170 gi|19424318|ref|NP_598290.1| & $\begin{array}{l}\text { enoyl-Coenzyme A, hydratase/3-hydroxyacyl Coenzyme A } \\
\text { dehydrogenase }\end{array}$ & 0.166 & 0.029 & 10 & 0.223 & 0.034 & 11 & 0.032 & 1.343 & 0.001 \\
\hline 171 gi|13928974|ref|NP_113886.1| & ribophorin 2; ribophorin II & 0.815 & 0.123 & 11 & 1.100 & 0.258 & 8 & 0.191 & 1.350 & 0.005 \\
\hline 172 gi|27690197|ref|XP_221062.1| & $\begin{array}{l}\text { similar to Macrophage migration inhibitory factor (MIF) } \\
\text { (Phenylpyruvate tautomerase) (Glutathione-binding } 13 \mathrm{kDa} \text { protein) }\end{array}$ & 0.316 & 0.123 & 3 & 0.428 & NA & 1 & 0.123 & 1.354 & 0.255 \\
\hline 173 gi|27687455|ref|XP_226755.1| & similar to cytoplasmic beta-actin & 0.693 & 0.196 & 5 & 0.946 & 0.247 & 3 & 0.214 & 1.365 & 0.157 \\
\hline $174 \mathrm{gi} \mid 13162347$ |ref|NP_077067.1| & adrenodoxin reductase & 0.492 & 0.028 & 2 & 0.672 & 0.148 & 3 & 0.122 & 1.366 & 0.204 \\
\hline 175 gi|6978543|ref|NP_036636.1| & $\begin{array}{l}\text { ATPase, } \mathrm{Na}+\mathrm{K}+\text { transporting, alpha } 1 ; \text { ATPase, } \mathrm{Na}+\mathrm{K}+ \\
\text { transporting, alpha } 1 \text { polypeptide }\end{array}$ & 0.419 & 0.100 & 4 & 0.573 & 0.208 & 6 & 0.175 & 1.368 & 0.211 \\
\hline 176 gi|27672918|ref|XP_213364.1| & similar to Eukaryotic initiation factor 4A-I (elF-4A-I) (elF4A-I) & 0.477 & 0.054 & 16 & 0.653 & 0.121 & 9 & 0.084 & 1.369 & $<0.001$ \\
\hline 177 gi|13027475|ref|NP_076492.1| & SECIS binding protein 2 & 34.011 & 25.271 & 2 & 46.631 & NA & 1 & 25.271 & 1.371 & 0.609 \\
\hline 178 gi|6981236|ref|NP_037326.1| & myosin, heavy polypeptide 9, non-muscle & 0.554 & 0.136 & 2 & 0.761 & 0.229 & 3 & 0.203 & 1.374 & 0.345 \\
\hline 179 gi|20302063|ref|NP_620239.1| & $\begin{array}{l}\text { aldo-keto reductase family 1, member D1 (delta 4-3-ketosteroid-5- } \\
\text { beta-reductase) }\end{array}$ & 0.261 & 0.067 & 8 & 0.359 & 0.063 & 5 & 0.066 & 1.375 & 0.024 \\
\hline 180 gi|13592067|ref|NP_112370.1| & ribosomal protein S9 & 0.468 & 0.083 & 11 & 0.645 & 0.047 & 3 & 0.078 & 1.378 & 0.005 \\
\hline 181 gi|6978747|ref|NP_036862.1| & cytochrome P450, subfamily IID2 & 1.319 & 0.523 & 63 & 1.820 & 0.594 & 27 & 0.545 & 1.380 & $<0.001$ \\
\hline 182 gi|9506375|ref|NP_062159.1| & $\begin{array}{l}\text { alcohol dehydrogenase 1; alcohol dehydrogenase (class I), alpha } \\
\text { polypeptide; alcohol dehydrogenase } 1 \text {, complex }\end{array}$ & 0.397 & 0.058 & 10 & 0.548 & 0.108 & 10 & 0.087 & 1.380 & 0.001 \\
\hline 183 gi|13591985|ref|NP_112313.1| & macrophage migration inhibitory factor & 0.350 & NA & 1 & 0.484 & 0.066 & 2 & 0.066 & 1.383 & 0.213 \\
\hline $184 \mathrm{gi}|20302049| \mathrm{ref}|\mathrm{NP} 620232.1|$ & diaphorase 1; Diaphorase (NADH) (cytochrome b-5 reductase) & 0.690 & 0.114 & 12 & 0.956 & 0.213 & 15 & 0.176 & 1.386 & 0.001 \\
\hline 185 gi|21955130|ref|NP_665713.1| & acyl-Coenzyme A oxidase 2 , branched chain & 0.601 & 0.100 & 3 & 0.837 & 0.092 & 4 & 0.095 & 1.393 & 0.023 \\
\hline 186 gi|15805031|ref|NP 284925.1| & eukaryotic translation elongation factor 1 alpha 2 & 0.342 & 0.089 & 13 & 0.479 & 0.094 & 9 & 0.091 & 1.401 & 0.002 \\
\hline 187 gil11968126|ref|NP $071981.1 \mid$ & cyclophilin B & 0.962 & 0.180 & 2 & 1.351 & NA & 1 & 0.180 & 1.404 & 0.201 \\
\hline 188 gi|6978813|ref|NP_036976.1| & $\begin{array}{l}\text { epoxide hydrolase 1; Epoxide hydrolase } 1 \text { (microsomal xenobiotic } \\
\text { hydrolase; Epoxide hydrolase } 1 \text { (microsomal xenobiotic hydrolase) }\end{array}$ & 0.599 & 0.106 & 27 & 0.842 & 0.141 & 20 & 0.122 & 1.406 & $<0.001$ \\
\hline 189 gi|13929196|ref|NP_114023.1| & beta-alanine-pyruvate aminotransferase & 0.227 & NA & 1 & 0.320 & 0.064 & 2 & 0.064 & 1.410 & 0.288 \\
\hline
\end{tabular}


190 gi|10120486|ref|NP_065415.1| glutathione S-transferase Yb4 gene

\begin{tabular}{|c|c|c|c|c|c|c|c|c|}
\hline 0.620 & 0.137 & 4 & 0.876 & 0.141 & 3 & 0.139 & 1.413 & 0.060 \\
\hline 2.527 & 0.743 & 10 & 3.572 & 1.507 & 14 & 1.252 & 1.414 & 0.056 \\
\hline 0.916 & NA & 1 & 1.295 & 0.662 & 2 & 0.662 & 1.414 & 0.567 \\
\hline 0.591 & 0.093 & 6 & 0.840 & NA & 1 & 0.093 & 1.421 & 0.001 \\
\hline 0.486 & 0.054 & 6 & 0.693 & 0.082 & 5 & 0.068 & 1.426 & 0.001 \\
\hline 0.585 & 0.150 & 12 & 0.836 & 0.208 & 18 & 0.187 & 1.429 & 0.001 \\
\hline 0.270 & 0.046 & 13 & 0.386 & 0.100 & 25 & 0.086 & 1.430 & $<0.001$ \\
\hline 0.466 & 0.141 & 11 & 0.667 & 0.105 & 10 & 0.125 & 1.431 & 0.002 \\
\hline 0.648 & 0.122 & 8 & 0.931 & 0.172 & 4 & 0.139 & 1.437 & 0.008 \\
\hline 1.120 & 0.305 & 13 & 1.615 & 0.346 & 12 & 0.325 & 1.442 & 0.001 \\
\hline 0.796 & 0.295 & 7 & 1.149 & 0.007 & 2 & 0.273 & 1.443 & 0.151 \\
\hline 0.822 & 0.262 & 4 & 1.188 & 0.972 & 4 & 0.712 & 1.445 & 0.495 \\
\hline 0.436 & 0.047 & 7 & 0.633 & 0.053 & 7 & 0.050 & 1.452 & $<0.001$ \\
\hline 0.474 & 0.072 & 17 & 0.689 & 0.099 & 10 & 0.083 & 1.454 & $<0.001$ \\
\hline 0.238 & 0.079 & 2 & 0.346 & 0.162 & 4 & 0.146 & 1.454 & 0.440 \\
\hline 0.927 & 0.200 & 26 & 1.351 & 0.367 & 25 & 0.294 & 1.457 & $<0.001$ \\
\hline 0.484 & 0.039 & 4 & 0.706 & 0.166 & 9 & 0.143 & 1.459 & 0.025 \\
\hline 0.296 & 0.064 & 4 & 0.433 & 0.073 & 3 & 0.068 & 1.463 & 0.046 \\
\hline 0.805 & 0.143 & 2 & 1.180 & 0.799 & 2 & 0.574 & 1.466 & 0.581 \\
\hline 0.779 & NA & 1 & 1.142 & 0.263 & 3 & 0.263 & 1.466 & 0.139 \\
\hline 0.601 & NA & 1 & 0.883 & 0.442 & 3 & 0.442 & 1.469 & 0.384 \\
\hline 0.682 & NA & 1 & 1.004 & 0.274 & 2 & 0.274 & 1.472 & 0.345 \\
\hline 0.753 & 0.133 & 6 & 1.111 & 0.140 & 5 & 0.136 & 1.475 & 0.002 \\
\hline 0.601 & 0.088 & 13 & 0.887 & 0.101 & 5 & 0.091 & 1.476 & $<0.001$ \\
\hline 1.350 & 0.536 & 10 & 1.993 & 0.539 & 17 & 0.538 & 1.476 & 0.006 \\
\hline 0.582 & NA & 1 & 0.860 & 0.139 & 2 & 0.139 & 1.478 & 0.216 \\
\hline 0.715 & 0.072 & 4 & 1.058 & 0.686 & 3 & 0.437 & 1.480 & 0.352 \\
\hline 0.492 & 0.399 & 5 & 0.729 & 0.116 & 8 & 0.258 & 1.482 & 0.135 \\
\hline 0.130 & 0.004 & 2 & 0.193 & 0.078 & 2 & 0.055 & 1.485 & 0.372 \\
\hline 0.693 & 0.098 & 11 & 1.030 & 0.167 & 22 & 0.148 & 1.486 & $<0.001$ \\
\hline 0.859 & 0.518 & 6 & 1.277 & 0.636 & 6 & 0.580 & 1.487 & 0.240 \\
\hline 0.630 & 0.204 & 5 & 0.937 & 0.197 & 10 & 0.199 & 1.487 & 0.015 \\
\hline 35.284 & 9.817 & 4 & 52.689 & NA & 1 & 9.817 & 1.493 & 0.038 \\
\hline 0.391 & NA & 1 & 0.589 & 0.074 & 5 & 0.074 & 1.506 & 0.004 \\
\hline 1.055 & 0.182 & 2 & 1.600 & NA & 1 & 0.182 & 1.517 & 0.148 \\
\hline 0.569 & 0.156 & 9 & 0.870 & 0.054 & 2 & 0.148 & 1.529 & 0.029 \\
\hline 0.736 & NA & 1 & 1.132 & 0.173 & 3 & 0.173 & 1.538 & 0.058 \\
\hline 0.506 & 0.126 & 12 & 0.779 & NA & 1 & 0.126 & 1.540 & $<0.001$ \\
\hline 0.981 & 0.188 & 22 & 1.513 & 0.303 & 30 & 0.261 & 1.542 & $<0.001$ \\
\hline 1.140 & 0.199 & 5 & 1.759 & 0.802 & 6 & 0.612 & 1.543 & 0.129 \\
\hline 0.501 & NA & 1 & 0.774 & 0.187 & 4 & 0.187 & 1.545 & 0.062 \\
\hline 0.354 & 0.173 & 4 & 0.547 & 0.148 & 8 & 0.156 & 1.545 & 0.071 \\
\hline 0.603 & 0.106 & 21 & 0.936 & 0.100 & 9 & 0.104 & 1.552 & $<0.001$ \\
\hline 0.543 & 0.049 & 17 & 0.844 & 0.206 & 6 & 0.109 & 1.554 & $<0.001$ \\
\hline
\end{tabular}

192 gi|27708678|ref|XP 231471.1| protein) [Homo sapiens]

192 gi|27708678|ref|XP 231471.1| similar to butyrate-induced transcript 1; hypothetical protein; butyrate-induced transcript 1 [Homo sapiens]

193 gi|27691290|ref|XP_227226.1| similar to 60S ribosomal protein L12

194 gi|8394009|ref|NP_058797.1| peptidylprolyl isomerase A (cyclophilin A)

195 gi|13162326|ref|NP_077057.1| bile acid CoA ligase

196 gi|15100179|ref|NP_150238.1| malate dehydrogenase-like enzyme

$197 \mathrm{gi}|25453414| \mathrm{ref}\left|\mathrm{NP} \_037289.1\right|$ arginosuccinate synthetase 1

198 gi|13994184|ref|NP_113937.1| glucosidase 1

199 gi|13929204|ref|NP_114027.1| arachidonic acid epoxygenase

200 gi|13929028|ref|NP 113919.1| aldehyde dehydrogenase family 3, subfamily A2; alcoho dehydrogenase family 3 , subfamily $A 2$

201 gi|9506703|ref|NP_062016.1| $\quad$ fibronectin 1

203 gi|27694244|ref|XP 214015.1| similar to UDP-glucuronosyltransferase 2B1 precursor, microsomal 0.47 (UDPGT) (UDPGTR-2)

204 gi|27658882|ref|XP_226351.1| similar to alanine aminotransferase 2; glutamic-pyruvate transaminase 2 [Homo sapiens]

205 gi|16758274|ref|NP_445964.1| peroxiredoxin 4

206 gi|27688923|ref|XP 213436.1| similar to ribosome-binding protein p34 - rat

207 gi|27668422|ref|XP 213262.1| similar to alpha globin

208 gi|27715583|ref|XP_216482.1| similar to RIKEN cDNA 0610040B21 [Mus musculus]

209 gi|27694804|ref|XP_215682.1| similar to glutathione-S-transferase, mu 2 [Mus musculus]

210 gi|13929194|ref|NP_114022.1| minoxidil sulfotransferase; aryl sulfotransferase IV; sulfotransferase family, cytosolic, 1A, phenol preferring: Phenol sulfotransferase 1a1

211 gi|27691358|ref|XP_227203.1| similar to DEAD/H box polypeptide 36 protein [Mus musculus]

212 gi|27673180|ref|XP_220576.1| similar to carcinoma related gene [Mus musculus]

213 gi|8392920|ref|NP_058830.1| arginase 1; arginase 1, liver

214 gi|21717659|ref|NP 579829.1| carboxylesterase 3

215 gi|9506523|ref|NP_062157.1| chondroitin sulfate proteoglycan 5; neuroglycan C

216 gi|20127395|ref|NP_446220.1| urate oxidase; uricase; urate oxidase 2 isoenzyme 1

219 gi|8393557|ref|NP_058929.1| 4-hydroxyphenylpyruvic acid dioxygenase

4-hydroxyphenylpyruvic acid dioxygenase 0.693

220 gi|8393322|ref|NP_059015.1| glucose regulated protein, 58 kDa; ER-60 protease; oxidoreductase ERp57

221 gi|21426797|ref|NP_653340.1| flavin containing monooxygenase

222 gil27689699|ref|XP 226986.1| similar to Ecotropic virus integration 1 site protein

223 gi|27717035|ref|XP 216669.1] similar to expressed sequence AL024210 [Mus musculus]

224 gi|13928740|ref|NP_113734.1| regucalcin

225 gi|19924087|ref|NP_612556.1| 3-alpha-hydroxysteroid dehydrogenase; steroid 3-alphadehydrogenase

226 gi|21489987|ref|NP 659553.1| chloride ion pump-associated $55 \mathrm{kDa}$ protein

227 gil16758920|ref|NP 446456.1| TBP-interacting protein 120A

228 gi|6981598|ref|NP_036793.1| steroid sulfatase

229 gi|13928768|ref|NP_113753.1| carboxylesterase 1

230 gi|6981682|ref|NP_037180.1| tocopherol transfer protein alpha

231 gi|20128770|ref|NP_569091.1| UDP glycosyltransferase 1 family, polypeptide A7

232 gi|16758646|ref|NP 446253.1] SEC14-like 2

233 gi|27715243|ref|XP_216452.1| similar to 24-dehydrocholesterol reductase; seladin-1; 3 betahydroxysterol delta 24 reductase [Homo sapiens]

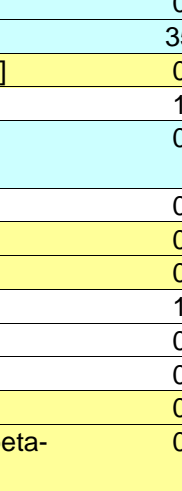


236 gi|11693158|ref|NP 071784.1| 7-dehydrocholesterol reductase

\begin{tabular}{lllllllll}
0.325 & 0.051 & 9 & 0.509 & 0.113 & 20 & 0.099 & 1.566 & $<0.001$ \\
\hline
\end{tabular}

$237 \mathrm{gi}|8393349|$ ref|NP_058877.1| fumarylacetoacetate hydrolase

238 gi|13399338|ref|NP_085075.1| cytochrome b5, outer mitochondrial membrane isoform

239 gi|27674996|ref|XP_214155.1| similar to purine-nucleoside phopshorylase [Mus musculus]

240 gil16758056|ref|NP 445791.1| acyl-Coenzyme A oxidase 3, pristanoyl

241 gi|25742754|ref|NP_036992.1| methionine adenosyltransferase I, alpha; S - adenosylmethionine synthetase

242 gi|27678390|ref|XP_222891.1| similar to migration inhibitory facto

243 gil17865351|ref|NP 446316.1| valosin-containing protein

similar to Microsomal triglyceride transfer protein, large subunit $\quad 0.480$ precursor

245 gi|19924039|ref|NP_612521.1| $\quad$ cytochrome P450 2c22

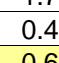

similar to dolichyl-di-phosphooligosaccharide-protein glycotransferase [Mus musculus]

248 gi|11968086|ref|NP 071955.1| $\quad$ ribosomal protein L4

249 gi|23463309|ref|NP_695226.1| UDP-glucuronosyltransferase 2B3 precursor, microsomal; liver 17 beta-hydroxysteroid UDP-glucuronosyltransferase

250 gi|17985949|ref|NP 150237.1| hemoglobin, beta

251 gi|25282419|ref|NP 742005.1| calnexin

\begin{tabular}{lll}
\hline 252 gi|11560006/ref|NP_071556.1| & L-gulono-gamma-lactone oxidase & 0.75 \\
\hline 253 gi|27673888|ref|XP_220650.1| & similar to carbohydrate kinase-like; RIKEN cDNA 4930431K22 gene & 0.50
\end{tabular} [Mus musculus]

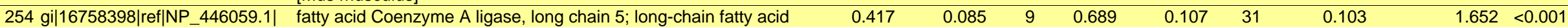
coenzyme A ligase 5

255 gi|27694423|ref|XP_223299.1| $\quad$ similar to expressed sequence Al788959; hypothetical protein $\quad 0.44$ MGC37823 [Mus musculus]

256 gi|19705453|ref|NP_599176.1| microsomal glutathione S-transferase 1

257 gi|13540663|ref|NP 110477.1| betaine-homocysteine methyltransferase

$258 \mathrm{gi|} 27658354|\mathrm{ref}| \mathrm{XP}$ 212835.1| similar to carboxylesterase isoenzyme

259 gil23463315|refINP 695225.1| cytochrome P450 2D1; debrisoquine 4-hydroxylase

gind dexylase

260 gi|11693172|ref|NP_071794.1| calreticulin

261 gi|27713820|ref|XP_232633.1| similar to ASPARTATE AMINOTRANSFERASE, MITOCHONDRIAL 0.32 PRECURSOR (TRANSAMINASE A) (GLUTAMATE

OXALOACETATE TRANSAMINASE-2)

262 gil27705988|ref|XP 228274.1 sphingosine-1-phosphate lyase 1

263 gi|6981594|ref|NP_037015.1| $\quad$ sulfotransferase, estrogen preferring; Estrogen sulfotransferase $\quad 4.107$

264 gi|19424302|ref|NP_598280.1| UDP-glucose glycoprotein: glucosyltransferase UGGT $\quad 0.645$

265 gi|27694223|ref|XP 223291.1| similar to UDP-glucuronosyltransferase 2B2 precursor, microsomal 1.321 (UDPGT) (3-hydroxyandrogen specific) (UDPGTR-4) (RLUG23)

\begin{tabular}{|c|c|c|c|c|c|c|c|c|c|c|}
\hline 266 gi|7106353|ref|NP_036732.1| & malic enzyme 1; Malic enzyme 1 , soluble & 0.055 & NA & 1 & 0.095 & 0.054 & 2 & 0.054 & 1.727 & 0.485 \\
\hline 267 gi|9506447|ref|NP_062165.1| & carbonic anhydrase 3 & 1.994 & 0.789 & 30 & 3.457 & 1.772 & 12 & 1.147 & 1.734 & 0.001 \\
\hline 268 gi|25742739|ref|NP_036952.1| & $\begin{array}{l}\text { fatty acid Coenzyme A ligase, long chain 2; Acyl CoA synthetase, } \\
\text { long chain }\end{array}$ & 0.680 & 0.098 & 6 & 1.184 & 0.199 & 15 & 0.178 & 1.741 & $<0.001$ \\
\hline 269 gi|8393499|ref|NP_058709.1| & $\begin{array}{l}\text { glutathione-S-transferase, alpha type2; Glutathione-S-transferase, } \\
\text { alpha type (Yc?) }\end{array}$ & 0.506 & 0.088 & 2 & 0.885 & 0.239 & 4 & 0.212 & 1.749 & 0.107 \\
\hline 270 gi|8394364|ref|NP_058895.1| & signal sequence receptor 4 ; signal sequence receptor, delta & 0.500 & 0.049 & 5 & 0.875 & 0.114 & 9 & 0.097 & 1.750 & $<0.001$ \\
\hline $271 \mathrm{gi}|20302024|$ ref|NP_620222.1| & oxygen regulated protein $(150 \mathrm{kD})$ & 0.847 & 0.082 & 11 & 1.495 & 0.262 & 17 & 0.212 & 1.765 & $<0.001$ \\
\hline 272 gi|6981392|ref|NP_037142.1| & $\begin{array}{l}\text { Protein kinase, AMP-activated, gamma } 1 \text { non-catalytic subunit; } \\
\text { Protein kinase, AMP-activated, gamma }\end{array}$ & 0.537 & 0.155 & 4 & 0.951 & NA & 1 & 0.155 & 1.771 & 0.013 \\
\hline 273 gi|8392839|ref|NP_058683.1| & ATP citrate lyase & 0.149 & 0.068 & 8 & 0.273 & 0.059 & 11 & 0.063 & 1.832 & 0.00 \\
\hline
\end{tabular}




\begin{tabular}{|c|c|c|c|c|c|c|c|c|c|c|}
\hline 274 gi|6978607|ref|NP_036652.1| & catalase & 0.471 & 0.087 & 26 & 0.867 & 0.179 & 30 & 0.144 & 1.841 & $<0.001$ \\
\hline 275 gi|6981324|ref|NP_037130.1| & protein disulfide isomerase (Prolyl 4-hydroxylase, beta polypeptide) & 0.737 & 0.202 & 12 & 1.367 & 0.301 & 16 & 0.264 & 1.855 & $<0.001$ \\
\hline 276 gi|6981486|ref|NP_037199.1| & ribophorin I & 0.575 & 0.149 & 17 & 1.082 & 0.182 & 11 & 0.162 & 1.882 & $<0.001$ \\
\hline 277 gi|16758712|ref|NP_446301.1| & $\begin{array}{l}\text { protein disulfide isomerase related protein (calcium-binding protein, } \\
\text { intestinal-related) }\end{array}$ & 0.693 & 0.262 & 2 & 1.308 & 0.343 & 2 & 0.305 & 1.887 & 0.181 \\
\hline 278 gi|8394149|ref|NP_059036.1| & acyl-coA oxidase & 0.309 & 0.156 & 5 & 0.588 & NA & 1 & 0.156 & 1.903 & 0.016 \\
\hline 279 gi|27706720|ref|XP_231135.1| & similar to hypothetical protein FLJ23816 [Homo sapiens] & 0.442 & 0.001 & 2 & 0.845 & 0.143 & 3 & 0.117 & 1.912 & 0.032 \\
\hline 280 gi|8392962|ref|NP_058996.1| & $\begin{array}{l}\text { bile acid-Coenzyme A: amino acid } \mathrm{N} \text {-acyltransferase; bile acid- } \\
\text { Coenzyme A dehydrogenase: amino acid n-acyltransferase }\end{array}$ & 0.591 & 0.276 & 7 & 1.143 & 0.371 & 12 & 0.341 & 1.934 & 0.003 \\
\hline $281 \mathrm{gi}|27693698|$ ref|XP_223244.1| & similar to Ser/Thr kinase KKIAMRE-gamma [Mus musculus] & 0.388 & NA & 1 & 0.764 & 0.177 & 3 & 0.177 & 1.969 & 0.067 \\
\hline 282 gi|9506529|ref|NP_062057.1| & $\begin{array}{l}\text { Cytochrome P450, subfamily IIC (mephenytoin 4-hydroxylase); } \\
\text { cytochrome P450, 2c29 }\end{array}$ & 1.761 & 0.339 & 26 & 3.496 & 1.001 & 43 & 0.819 & 1.985 & $<0.001$ \\
\hline 283 gi|8393215|ref|NP_058770.1| & CTL target antigen & 1.050 & 0.569 & 4 & 2.117 & NA & 1 & 0.569 & 2.016 & 0.033 \\
\hline 284 gi|13592047|ref|NP_112359.1| & aminopeptidase B & 0.566 & 0.080 & 12 & 1.142 & 0.436 & 5 & 0.235 & 2.018 & $<0.001$ \\
\hline 285 gi|13242293|ref|NP_077362.1| & 3-hydroxy-3-methylglutaryl CoA lyase & 0.308 & NA & 1 & 0.641 & 0.436 & 2 & 0.436 & 2.081 & 0.475 \\
\hline 286 gi|27732483|ref|XP_230613.1| & $\begin{array}{l}\text { similar to hydroxyacid oxidase } 1 \text {, liver; glycolate oxidase } 1 \text { [Mus } \\
\text { musculus] }\end{array}$ & 0.519 & NA & 1 & 1.084 & 0.152 & 6 & 0.152 & 2.089 & $<0.001$ \\
\hline 287 gi|27702070|ref|XP_215769.1| & similar to mitochondrial carrier homolog 2 [Mus musculus] & 0.113 & NA & 1 & 0.237 & 0.030 & 3 & 0.030 & 2.097 & 0.019 \\
\hline 288 gi|8394334|ref|NP_058748.1| & sorbitol dehydrogenase & 0.401 & 0.142 & 3 & 0.853 & 0.426 & 2 & 0.272 & 2.127 & 0.166 \\
\hline 289 gi|27705028|ref|XP_215371.1| & similar to ubiquitin conjugating enzyme 7 [Mus musculus] & 0.688 & 0.155 & 3 & 1.488 & 0.134 & 2 & 0.148 & 2.163 & 0.010 \\
\hline 290 gi|19924031|ref|NP_612517.1| & sterol carrier protein 2, liver & 0.203 & NA & 1 & 0.443 & 0.276 & 3 & 0.276 & 2.182 & 0.271 \\
\hline $291 \mathrm{gi|} 27715247 \mid$ ref|XP_233266.1| & similar to hypothetical protein FLJ20619 [Homo sapiens] & 0.021 & 0.011 & 2 & 0.046 & NA & 1 & 0.011 & 2.190 & 0.192 \\
\hline 292 gi|19424278|ref|NP_598267.1| & $\mathrm{N}$-myc downstream-regulated gene 2 & 0.264 & 0.045 & 2 & 0.579 & 0.333 & 2 & 0.238 & 2.193 & 0.316 \\
\hline 293 gi|8393296|ref|NP_058941.1| & eukaryotic translation elongation factor 2 & 0.350 & 0.083 & 6 & 0.768 & 0.160 & 12 & 0.141 & 2.194 & $<0.001$ \\
\hline 294 gi|27677480|ref|XP_214127.1| & UDP-glucuronosyltransferase 2 family, member 5 & 1.232 & 0.162 & 4 & 2.808 & 3.479 & 5 & 2.632 & 2.279 & 0.402 \\
\hline 295 gi|27712702|ref|XP_216227.1| & similar to forkhead-related transcription factor 1C [Mus musculus] & 1.851 & NA & 1 & 4.508 & 0.740 & 2 & 0.740 & 2.435 & 0.124 \\
\hline 296 gi|6978741|ref|NP_036825.1| & $\begin{array}{l}\text { cytochrome P450, subfamily } 2 \text { A, polypeptide } 1 \text {; Cytochrome P450 } \\
\text { IIA2 }\end{array}$ & 0.353 & 0.129 & 2 & 0.863 & 0.561 & 2 & 0.407 & 2.445 & 0.337 \\
\hline 297 gi|19705467|ref|NP_599196.1| & cytochrome P450 monooxygenase CYP2T1 & 1.409 & 0.948 & 3 & 3.492 & NA & 1 & 0.948 & 2.478 & 0.063 \\
\hline 298 gi|14861868|ref|NP_149090.1| & $\begin{array}{l}\text { Osteotesticular phosphatase; protein tyrosine phosphatase, } \\
\text { receptor type, W }\end{array}$ & 0.499 & 0.377 & 6 & 1.255 & 0.251 & 5 & 0.327 & 2.515 & 0.004 \\
\hline 299 gi|27692979|ref|XP_223202.1| & similar to Elongation factor 2 (EF-2) & 0.343 & 0.061 & 2 & 0.874 & 0.128 & 6 & 0.119 & 2.548 & 0.002 \\
\hline 300 gi|6981050|ref|NP_036716.1| & $\begin{array}{l}\text { hydroxy-delta-5-steroid dehydrogenase, } 3 \text { beta- and steroid delta- } \\
\text { isomerase; Hydroxy-delta-5-steroid dehydrogenase, } 3 \text { beta- and } \\
\text { steroid delta-isomerase }\end{array}$ & 0.735 & NA & 1 & 1.902 & 0.050 & 2 & 0.050 & 2.588 & 0.019 \\
\hline 301 gi|6978695|ref|NP_036664.1| & ceruloplasmin; Ceruloplasmin (ferroxidase) & 0.282 & NA & 1 & 0.780 & 0.339 & 2 & 0.339 & 2.766 & 0.286 \\
\hline 302 gi|16758486|ref|NP_446126.1| & phytanoyl-CoA hydroxylase (Refsum disease) & 0.298 & 0.170 & 4 & 1.153 & NA & 1 & 0.170 & 3.869 & 0.002 \\
\hline 303 gi|27665054|ref|XP_213577.1| & $\begin{array}{l}\text { similar to Ubiquitin-conjugating enzyme E2-18 kDa UbcH7 (Ubiquitin } \\
\text { protein ligase) (Ubiquitin carrier protein) (UbcM4) (E2-F1) (L-UBC) }\end{array}$ & 0.572 & 0.255 & 2 & 2.520 & NA & 1 & 0.255 & 4.406 & 0.059 \\
\hline 304 gi|27714771|ref|XP_233065.1| & $\begin{array}{l}\text { similar to protein tyrosine phosphatase, receptor type, D isoform } 3 \\
\text { precursor; protein tyrosine phosphatase, receptor type, delta } \\
\text { polypeptide; protein tyrosine phosphatase delta [Homo sapiens] }\end{array}$ & 9.297 & 4.117 & 3 & 45.315 & 18.274 & 2 & 11.073 & 4.874 & 0.038 \\
\hline 305 gi|9506591|ref|NP_062111.1| & farnesyl diphosphate farnesyl transferase 1 & 0.393 & 0.069 & 2 & 2.367 & NA & 1 & 0.069 & 6.023 & 0.016 \\
\hline 306 gi|20806131|ref|NP_620793.1| & CTD-binding SR-like protein rA9 & 0.105 & NA & 1 & 0.639 & 0.324 & 2 & 0.324 & 6.086 & 0.258 \\
\hline 307 gi|12621132|ref|NP_075243.1| & MEGF1 & 0.558 & 0.097 & 2 & 4.483 & 3.636 & 2 & 2.572 & 8.034 & 0.267 \\
\hline 308 gi|6978751|ref|NP_037073.1| & $\begin{array}{l}\text { cytochrome P450, subfamily 51; Cytochrom P450 Lanosterol } 14 \\
\text { alpha-demethylase }\end{array}$ & 0.052 & NA & 1 & 1.102 & 0.525 & 3 & 0.525 & 21.192 & 0.074 \\
\hline 309 gi|9845251|ref|NP_063971.1| & rapamycin and FKBP12 target-1 protein & 0.006 & 0.003 & 2 & 0.210 & 0.108 & 2 & 0.076 & 35.000 & 0.116 \\
\hline 310 gi|12711694|ref|NP_075413.1| & $\begin{array}{l}\text { dynein, cytoplasmic, heavy chain 2; cytoplasmic dynein heavy chain } \\
2\end{array}$ & 0.396 & 0.238 & 2 & 17.596 & 16.070 & 3 & 13.122 & 44.434 & 0.247 \\
\hline
\end{tabular}

